\title{
How do Parity Violating Weak Nuclear Interactions Influence Rovibrational Frequencies in Chiral Molecules?
}

\author{
By Martin Quack* and Jürgen Stohner \\ Laboratorium für Physikalische Chemie, ETH Zürich (Zentrum), CH-8092 Zürich, \\ Switzerland
}

Dedicated to Prof. Dr. Dr. h.c. mult. H. Gg. Wagner on the occasion of his 70th birthday

(Received February 4, 2000; accepted February 10, 2000)

\author{
Infrared Spectroscopy / Chiral Molecules / Parity Violation / \\ Electroweak Quantum Chemistry / Vibrational Spectra / \\ Rotational Spectra
}

\begin{abstract}
We outline the general theory as well as various approximations to the accurate calculation of vibrational and rotational transition frequency shifts between enantiomers of chiral molecules due to the parity violating weak nuclear interaction. The calculation of the effective parity violating potentials as a function of molecular geometry is mainly based on our recent, accurate Multiconfiguration-Linear Response approach (MC-LR, RPA and CASSCF, Berger and Quack, J. Chem. Phys. 112 (2000) 3148), which has been shown to lead to order of magnitude increases compared to early Restricted Hartree Fock (RHF) approaches. We present in some detail both the harmonic and diagonal anharmonic approach to parity violating vibrational frequency shifts. In these approaches the parity violating potentials are calculated as a function of all vibrational reduced normal coordinates $q_{i}$ with the relevant matrix elements beeing obtained from harmonic and anharmonic vibrational wavefunctions. We also compare the adiabatic and reverse adiabatic harmonic approximation. In addition to the general exact theory several approximate approaches are introduced for the calculation of parity violating structural differences between enantiomers resulting in the corresponding changes of rotational transition frequencies. Results are presented for the chiral molecule $\mathrm{CHBrClF}$. The predicted relative vibrational frequency shifts $\Delta_{\mathrm{pv}} \omega_{i} / \omega_{i}$ are shown to depend strongly on the vibrational mode and the level of calculation (RHF, RPA, CASSCF) but in all cases are on the order of $10^{-(16 \pm 1)}$, much smaller than all previous experimental tests could detect. The predicted relative rotational frequency shifts fall in a similar range. We discuss consequences of our predictions for various possible experiments on molecular parity violation.
\end{abstract}

* Corresponding author. E-mail: quack@ir.phys.chem.ethz.ch 


\section{Introduction}

Parity violation in nuclear physics was established in 1957 [1, 2] and the history as well as the unified electroweak theory [3-5] has been reviewed occasionally [6, 7]. Also, the consequences of parity violation in atomic physics and spectroscopy have been explored and reviewed [8, 9]. However, perhaps the most interesting consequence results in molecular physics, where parity violation leads to energy differences $\Delta_{\mathrm{pv}} E$ between the left and right handed enantiomers of chiral molecules. From early on, the possible relationship of this energy difference and the preferred selection of L-aminoacids and D-sugars has been discussed [10-16]. Possible experiments for a measurement of $\Delta_{\mathrm{pv}} E$ have been proposed [17] and these aspects have been reviewed [16], including extension to more general fundamental symmetry violations in molecular physics [18].

From the theoretical side an important development was the introduction of quantitative calculations of $\Delta_{\mathrm{pv}} E$ based on quantum chemical techniques and perturbation theory [19-26]. There has recently been a renewed interest in improving upon these early calculations based on a Single Determinant Excitation-Restricted Hartree Fock (SDE-RHF) framework because of the discovery $[27,28]$ of an order of magnitude increase in $\Delta_{\mathrm{pv}} E$, when going to a Configuration Interaction Singles excitation (CIS-RHF) approach, which has been reconfirmed more recently by independent computational techniques [29-33]. This discovery not only sheds new light on possible theoretical approaches but also is stimulating for developing experimental tests of molecular parity violation, which may be more easily detected than previously anticipated on the basis of the older results.

While a measurement of the parity violating energy difference $\Delta_{\mathrm{pv}} E$ between enantiomers would be the most significant test of parity violation in molecules [17], one may conceive also other experiments. One possibility consists in demonstrating frequency shifts $\Delta_{\mathrm{pv}} v=v^{(\mathrm{R})}-v^{(\mathrm{S})}$ for comparable spectroscopic transitions observed in the left and right handed enantiomers. Fig. 1 illustrates this approach in comparison with the measurement of $\Delta_{\mathrm{pv}} E$ itself.

In this energy level scheme of Fig. 1 the overall ground rovibronic state corresponds to the $(\mathrm{S})$ enantiomer, whereas the $(\mathrm{R})$ enantiomer has its ground state at an energy higher by $\Delta_{\mathrm{pv}} E$ (in the high barrier limit, see [16]). The measurement of $\Delta_{\mathrm{pv}} E$ relies on transitions to an excited state of well defined parity ("+" in Fig. 1, or at least sufficient excess parity), which connects optically to both $(\mathrm{R})$ and $(\mathrm{S})$ ground state levels [16, 17]. As a rule, by this technique one will obtain the maximum parity violating effect, which at the same time is a fundamental thermodynamic quantity, the enthalpy of the reaction at $0 \mathrm{~K}$

$$
\mathrm{S} \rightleftharpoons \mathrm{R} \quad \Delta_{\mathrm{pv}} E=\Delta_{\mathrm{r}} H_{0}^{\mathrm{o}} / N_{\mathrm{A}} .
$$

While this is quite small (for the example $\mathrm{CHBrClF}$ of the present paper 


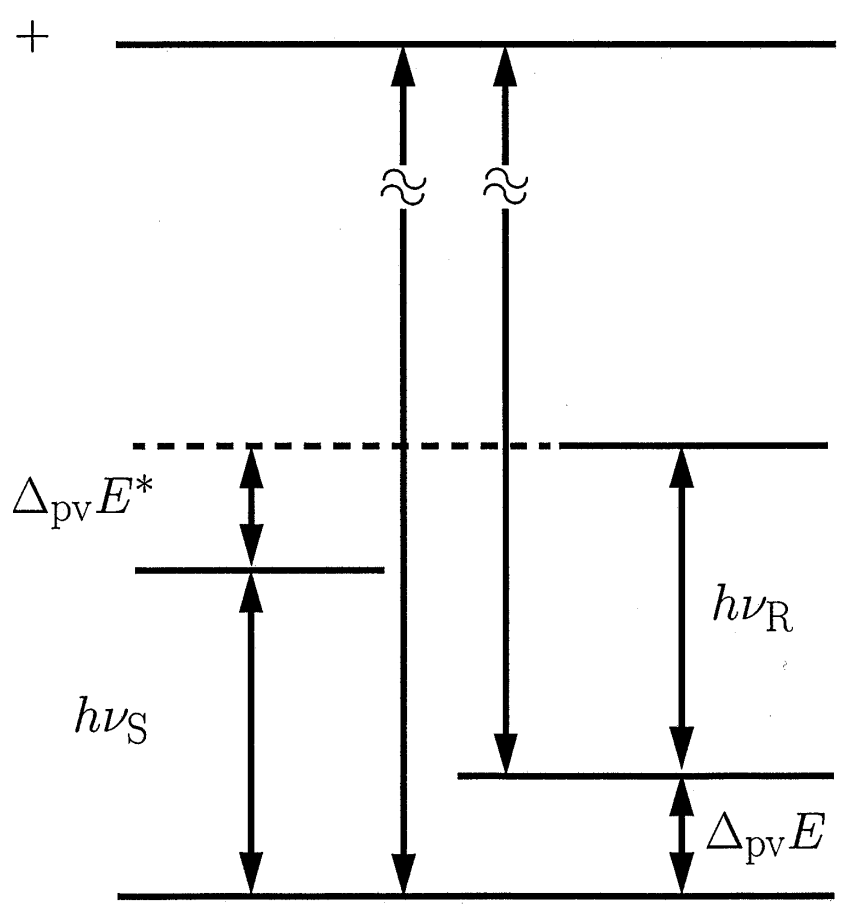

\section{(S)-CHBrClF}

(R)-CHBrClF

Fig. 1. Energy level scheme for a (R) enantiomer (right) and a (S) enantiomer (left) with an energy separation due to parity violation of $\Delta_{\mathrm{pv}} E$ (vibrational ground state) and $\Delta_{\mathrm{pv}} E^{*}$ (excited vibrational state) and the fundamental absorptions with transition energies of $h v_{\mathrm{R}}$ and $h v_{\mathrm{s}}$. We indicated transitions to an excited state with a definite parity, here positive. See text for discussion.

we calculate to be about $10^{-11} \mathrm{~J} \mathrm{~mol}^{-1}$ ), the other effects based on the second approach to molecular parity violation to be discussed now, will be even smaller, in general. This approach measures a transition frequency in either the (S) enantiomer or the (R) enantiomer and compares the two. Because as a rule the excited levels will be separated by $\Delta_{\mathrm{pv}} E^{*}$ very similar to $\Delta_{\mathrm{pv}} E$ the measurable $h\left|v^{(\mathrm{R})}-v^{(\mathrm{S})}\right|$ will be smaller than $\left|\Delta_{\mathrm{pv}} E\right|$, although exceptions to this rule are conceivable (the spectroscopic measurement of $\Delta_{\mathrm{pv}} E$ via a common intermediate level discussed above is an obvious exception). As we shall see below, $h\left|v^{(\mathrm{R})}-v^{(\mathrm{S})}\right|$ can be on the order of $10 \%$ of $\Delta_{\mathrm{pv}} E$ for vibrational transitions in the example CHBrClF. Measurements of $v^{(\mathrm{R})}-v^{(\mathrm{S})}$ have been proposed (and carried out) in a variety of spectral ranges from radiofrequency (NMR) [34], to microwave rotational spectra 


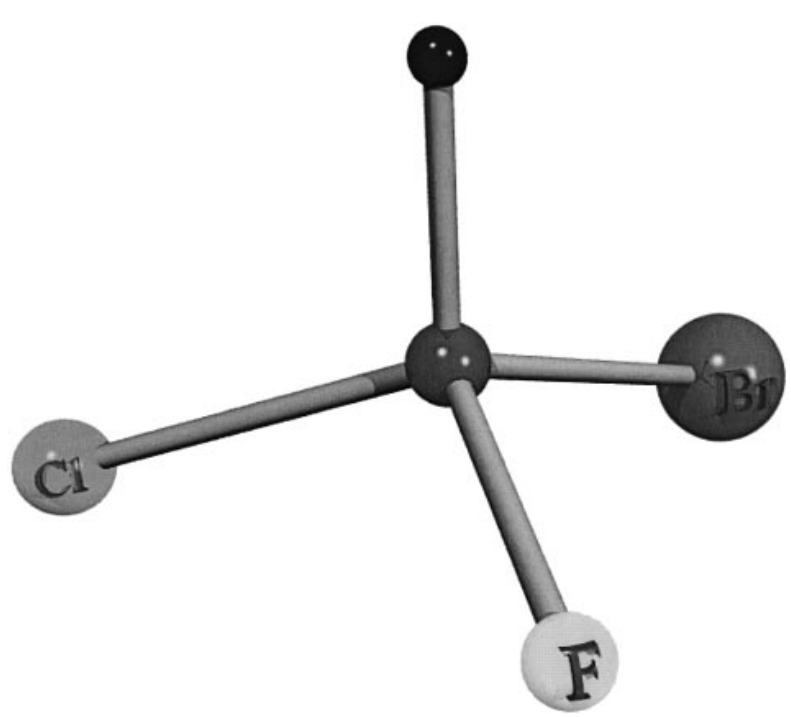

Fig. 2. Graphical representation of (R)-CHBrClF for the equilibrium structure. The $a b$ initio calculated internal coordinates [35] in the ordering of $r_{\mathrm{e}}^{0}(\mathrm{CH}), r_{\mathrm{e}}^{0}(\mathrm{CF}), r_{\mathrm{e}}^{0}(\mathrm{CCl})$, $r_{\mathrm{e}}^{0}(\mathrm{CBr}), \varphi_{\mathrm{e}}^{0}(\mathrm{HCF}), \varphi_{\mathrm{e}}^{0}(\mathrm{HCCl}), \varphi_{\mathrm{e}}^{0}(\mathrm{HCBr}), \varphi_{\mathrm{e}}^{0}(\mathrm{FCCl}), \varphi_{\mathrm{e}}^{0}(\mathrm{ClCBr})$ are as follows (bond lengths in $\underline{A}$, bond angles in degrees): 1.0807, 1.3609, 1.7529, 1.9220, 108.43, 108,51, 108.29, $109.02,113.38$. The $r_{\mathrm{e}}^{0}$ and $\varphi_{\mathrm{e}}^{0}$ correspond to the equilibrium geometry on the zero order (parity conserving) potential hypersurface (see section 2.5 ).

[35], infrared vibrational spectra [35-43] and perhaps $\gamma$-ray (Mössbauer) spectra [44]. While early experiments generally were limited to a relative accuracy $\Delta v / v \approx 10^{-6}$ to $10^{-8}$ [35-40], a most recent experiment led to an accuracy $\Delta v / v \approx 4 \times 10^{-13}$ and thus a corresponding upper limit $\Delta v / v<4 \times 10^{-13}$ in the $v_{4}$ fundamental of CHBrClF $[41,42]$.

In view of these experiments, accurate calculations of parity violating shifts in rovibrational spectra of chiral molecules are of interest. An early paper has provided already some very approximate estimates [25]. It seems, however, that our recent effort is the first serious attempt at an accurate calculation of these effects in polyatomic molecules. Preliminary results from the present work have been reported at scientific meetings [45, 46]. Subsequent recent work based on a relativistic Dirac Fock approach (to be compared with RHF at the nonrelativistic level) seems to confirm that relativistic corrections are relatively modest even for molecules involving $\mathrm{Br}$ and $\mathrm{Cl}$ atoms [47].

This paper presents first a detailed account of the various theoretical approaches and problems related to the calculation of rovibrational frequency shifts due to parity violation in chiral molecules. We then present numerical results for the molecule $\mathrm{CHBrClF}$ (Fig. 2). 
This molecule has been a prototype of chiral molecules for more than a century [48] and has been considered in the context of parity violation already in 1976 [39]. We have studied its rovibrational high resolution spectrum for almost a decade (see [35-38] and references cited therein) and have achieved the first rovibrational analysis of an infrared fundamental for any chiral molecule [35]. Based on our analysis of $v_{4}$, spectra at ultrahigh resolution taken by Daussy et al. [41, 42] on this band led to the most stringent bound for $h\left|v^{(\mathrm{R})}-v^{(\mathrm{S})}\right|$ so far available.

\section{Theory}

\subsection{Calculation of parity violating potentials in polyatomic molecules}

A simple term in the molecular Hamiltonian that transforms odd under parity and thus leads to parity violating potentials is given by

$$
\hat{H}_{\mathrm{pv}}=-\frac{G_{\mathrm{F}}}{2 \sqrt{2} m_{\mathrm{e}} c} \cdot \sum_{i, a}\left(N_{a}-Z_{a}\left(1-\sin ^{2}\left(\theta_{\mathrm{w}}\right)\right)\right) \hat{\vec{s}}_{i} \cdot\left[\hat{\vec{p}}_{i}, \delta^{3}\left(\vec{r}_{i}-\vec{r}_{a}\right)\right]_{+}
$$

where $G_{\mathrm{F}}$ is Fermi's coupling constant, $m_{\mathrm{e}}$ : electron mass, $c$ : velocity of light, $Z_{a}$ : nuclear charge of nucleus $a, N_{a}$ : neutron number of nucleus $a$, $\theta_{w}$ : Weinberg angle $\sin ^{2}\left(\theta_{\mathrm{w}}\right) \approx 0.224(19)$ [49] (in the computations we still used the somewhat older value 0.2319 [27-30]), $\delta^{3}\left(\vec{r}_{i}-\vec{r}_{a}\right)$ : Dirac's delta distribution, $\hat{s}_{i}$ : spin operator of electron $i, \hat{\vec{p}}_{i}$ : momentum operator of electron $i$, and $[\ldots]_{+}$denotes an anticommutator $[20,22,28,50]$. The anticommutator is defined through its action on a wavefunction to its right by considering the chain rule. The three dimensional Dirac delta distribution is a product of three one dimensional Dirac delta distributions [51] and applied to Eq. (2) we obtain together with

$$
\begin{gathered}
\delta^{3}\left(\vec{r}_{i}-\vec{r}_{a}\right)=\delta\left(x_{i}-x_{a}\right) \delta\left(y_{i}-y_{a}\right) \delta\left(z_{i}-z_{a}\right) \\
\int f\left(x_{i}\right) \frac{\partial^{n}}{\partial x_{a}^{n}} \delta\left(x_{i}-x_{a}\right) \mathrm{d} x_{i}=(-1)^{n} \frac{\partial^{n}}{\partial x_{a}^{n}} f\left(x_{a}\right)
\end{gathered}
$$

for a matrix element of the anticommutator (in $\mathrm{x}$, e.g.)

$$
\left\langle\Psi_{a}\left|\left[p_{x_{i}}, \delta\left(x_{i}-x_{a}\right)\right]_{+}\right| \Psi_{b}\right\rangle=+i\left(\left.\frac{\partial \Psi_{a}}{\partial x_{i}} \cdot \Psi_{b}\right|_{x_{i}=x_{a}}-\left.\Psi_{a} \cdot \frac{\partial \Psi_{b}}{\partial x_{i}}\right|_{x_{i}=x_{a}}\right) .
$$

Spin and spatial coordinates are completely separable. Eq. (5) shows that the parity violating contribution is related to the gradient of the wavefunction of the electron at the position of the nucleus (somewhat similar to the Fermi contact interaction in magnetic resonance [52], which is related to the square modulus of the wave function at the position of the nucleus). 
An estimate of the parity violating potential $V_{\mathrm{pw}}$ giving rise to energy difference between left- and right-handed enantiomers of a chiral molecule can be obtained from a sum-over-states expression (see [28], and refs. therein)

$$
V_{\mathrm{pv}}=2 \cdot \operatorname{Re} \sum_{n} \frac{\left\langle{ }^{1} \Psi_{0}\left|\hat{H}_{\mathrm{so}}\right|{ }^{3} \Psi_{n}\right\rangle \cdot\left\langle{ }^{3} \Psi_{n}\left|\hat{H}_{\mathrm{pv}}\right|{ }^{1} \Psi_{0}\right\rangle}{{ }^{1} E_{0}-{ }^{3} E_{n}} .
$$

${ }^{1} \Psi_{0}$ denotes the singlet electronic ground state wavefunction and ${ }^{3} \Psi_{n}$ the $n^{\text {th }}$ electronically excited triplet state wavefunction and ${ }^{1} E_{0},{ }^{3} E_{n}$ the corresponding energies. The matrix elements of the spin-orbit operator $\hat{H}_{\text {so }}$ (see e.g. $[28,30,53])$ and $\hat{H}_{\mathrm{pv}}$ are evaluated at cartesian geometries derived from a set of suitable non-redundant internal or reduced dimensionless normal coordinates (see below). The matrix elements for the spin-orbit and the parity-violating part are evaluated in a Gaussian-type atomic orbital basis and transformed to the molecular orbital basis set with the transformation matrix evaluated $a b$ initio, e.g. with Gaussian 94 [54] in the restricted Hartree-Fock (RHF) approximation or configuration interaction with singly excited determinants (CIS) along the lines described in [28]. Due to convergence problems of the sum-over-states expression, especially when CIS is used, we implemented a new approach to calculate $V_{\mathrm{pv}}$ in the framework of the multi-configurational linear-response (MC-LR) approximation [30] making use of DALTON $[55,56]$. DALTON allows to select several highquality wavefunctions for which the linear response equations can be solved; $E_{\mathrm{pv}}$ is obtained from the linear response of $H_{\mathrm{pv}}$ to the perturbation $H_{\mathrm{SO}}$ at zero frequency [30]. Besides RHF we use in the present work the random-phase approximation (RPA) and a MC-LR version of the complete active space self-consistent field (CASSCF) approach. More details and a description and discussion of approximations made to evaluate $V_{\mathrm{pv}}$ in our work can be found elsewhere [27-30].

\subsection{General theory of rovibrational frequency shifts in polyatomic molecules due to parity violation}

The influence of the parity violating potential on the vibrational and rotational frequencies can be determined once the potential $V_{\mathrm{pv}}$ is known in some suitable non-redundant set of $3 N-6$ coordinates, where $N$ is the number of atoms in the molecule. Suitable sets of coordinates are internal coordinates such as bond-lengths and bond-angles or normal coordinates. The latter have the distinct advantage of constituting a rectilinear coordinate system which offers considerable simplifications for the formulation of the vibrational Hamiltonian. A detailed discussion of the different choice of coordinate systems can be found in [36-38, 57-59].

As described in detail in our earlier work on the evaluation of multidimensional anharmonic potential energy surfaces for molecules with an 
isolated $\mathrm{CH}$ chromophore [57, 60, 61], dimensionless reduced normal coordinates $q_{j}$ ( $j$ from 1 to $3 N-6$ ) are a good choice for treating rovibrational levels of strongly bound molecules at not too high excitations. In the case of $\mathrm{N}$-atomic molecules one set of values for all normal coordinates corresponds to a set of $3 \mathrm{~N}$ cartesian coordinates for the atoms (implying a convention for the center of mass position and rotation). We obtain reduced normal coordinates $q_{j}$ from the diagonalization of the cartesian massweighted force constant matrix $\boldsymbol{F}^{(\boldsymbol{x}, \boldsymbol{m})}$

$$
q_{j}=\gamma_{j}^{1 / 2} \cdot \sum_{n} l_{n j} \cdot m_{n}^{1 / 2} \cdot x_{n}
$$

with $\gamma_{j}=\sqrt{\omega_{j}} / \hbar$ where $l_{n j}$ are the eigenvectors and $\omega_{j}$ are related to the eigenvalues; $m_{n}$ are the atomic masses and $x_{n}$ the atomic cartesian coordinates. We determine $V_{\mathrm{pv}}(\vec{q})$ along the reduced normal coordinates from the cartesian displacements [58]

$$
\Delta x_{n}^{(j)}=\gamma_{j}^{-1 / 2} \cdot l_{n j} \cdot m_{n}^{-1 / 2} \cdot \Delta q_{j}
$$

which define new cartesian geometries for elongation along $q_{j}$. These are then used to calculate $V_{\mathrm{pv}}(\vec{q})$ as described in the previous section.

The complete vibrational Hamiltonian can be written as

$$
\hat{H}(\hat{\bar{p}}, \vec{q})=\hat{T}(\hat{\bar{p}}, \vec{q})+V(\vec{q})+\hat{H}_{\mathrm{pv}}(\vec{q})=\hat{H}^{0}(\hat{\vec{p}}, \vec{q})+V_{\mathrm{pv}}(\vec{q})
$$

where $\hat{T}$ and $V$ are the kinetic and potential energy operators, and $V_{\mathrm{pv}}$ denotes the very small perturbation to the zeroth order Hamiltonian $\hat{H}^{0}$ due to parity violation (see Eq. (2)). The coordinate vector $\vec{q}$ has components $\left\{q_{i}\right\}$, $i=1 \cdots 3 N-6$; the $\hat{\vec{p}}_{i}$ are the conjugate momenta. The solution of the zeroth order Schrödinger equation

$$
\hat{H}^{0}\left|\phi_{n}\right\rangle=E_{n}^{0}\left|\phi_{n}\right\rangle
$$

provides the $n^{\text {th }}$ eigenvalue $E_{n}^{0}$ and the corresponding eigenfunction $\left|\phi_{n}\right\rangle$. If $\left|\Psi_{n}\right\rangle$ denotes the eigenfunction of $\hat{H}$,

$$
\hat{H}\left|\Psi_{n}\right\rangle=E_{n}\left|\Psi_{n}\right\rangle
$$

in general this needs now diagonalization of the full Hamiltonian. To a good approximation, the first order correction to the zeroth order energy, $E_{n}^{0}$, is given by

$$
E_{n}^{(1)} \approx\left\langle\phi_{n}\left|V_{\mathrm{pv}}\right| \phi_{n}\right\rangle \text {. }
$$

For a given enantiomer, the energy shift due to the parity violating interaction is given by

$$
E_{n}-E_{n}^{0} \approx\left\langle\phi_{n}\left|V_{\mathrm{pv}}\right| \phi_{n}\right\rangle \text {. }
$$

Correspondingly, the energy difference between enantiomers $\mathrm{R}$ and $\mathrm{S}$ is

$$
\Delta_{\mathrm{pv}} E=E_{n}^{(\mathrm{R})}-E_{n}^{\mathrm{O}(\mathrm{R})}-\left(E_{n}^{(\mathrm{S})}-E_{n}^{\mathrm{o}(\mathrm{S})}\right) \approx 2\left\langle\phi_{n}\left|V_{\mathrm{pv}}\right| \phi_{n}\right\rangle .
$$

Since the magnitude of the parity violating perturbation is very small, diagonal perturbation theory is expected to provide good estimates for the fre- 
quency shifts as long as they remain small with respect to the zeroth order level separations. In principle, the matrix elements in Eq. (13) is to be calculated with a fully anharmonically coupled multi-dimensional vibrational (or more generally rovibrational) wavefunction $\phi_{n}$. In practice some further approximations are useful.

\subsection{Separable harmonic and anharmonic approximation for adiabatic vibrational frequency shifts}

The multi-dimensional integral in Eq. (13) needs in a first stage the solution of the multidimensional anharmonic rovibrational problem with the Hamiltonian $\hat{H}^{0}$. Even with separation of rotation and vibration this type of problem has been solved exactly only for molecular problems with a maximum of six to nine vibrational degrees of freedom (see for example [62, 63]). The formulation of a fully coupled $3 N-6$ dimensional anharmonic potential energy hypersurface for a chiral molecule represents a substantial task (see for example the formulation of methane potentials in [64]) and so would the formulation of a fully coupled $V_{\mathrm{pv}}$ for $3 N-6$ degrees of freedom in chiral molecules. It seems natural to start out with a separable normal mode approximation for $\hat{H}^{0}$, and thus

$$
\begin{aligned}
\hat{H}^{0} & =\sum_{j=1}^{3 N-6} \hat{H}_{j}^{0}\left(p_{j}, q_{j}\right) \\
E_{n}^{0} & =\sum_{j=1}^{3 N-6} E_{n, j}^{0} \\
\phi_{n} & =\prod_{j=1}^{3 N-6} \varphi_{n, j}\left(q_{j}\right)
\end{aligned}
$$

where the sums (or the product) extend over all normal modes. In the strictly harmonic approximation, the $\varphi_{n, j}$ are harmonic oscillator functions. More generally one may solve the one-dimensional anharmonic Schrödinger equation on a grid [65] for each normal coordinate and obtain separable one dimensional anharmonic zero order energies $E_{n, j}^{0}$ and wavefunctions $\varphi_{n, j}\left(q_{j}\right)$ in each vibrational mode. The first order parity violating energy shift for each vibrational mode is then given by

$$
E_{n, j}-E_{n, j}^{0} \approx\left\langle\varphi_{n, j}\left|V_{\mathrm{pv}}\left(q_{j}\right)\right| \varphi_{n, j}\right\rangle .
$$

In a further step one may include the most important vibrational anharmonic couplings, which leads typically to coupled three or four dimensional problems, which are difficult but still manageable and can be solved exactly on $3 \mathrm{D}$ and $4 \mathrm{D}$ grids $[36,66,67]$.

In the present paper we restrict our attention to the one dimensional approximations. The relative vibrational frequency shift for a vibrational transition in mode $j$ between two enantiomers is then given by 


$$
\Delta_{\mathrm{pv}} v_{j}^{\mathrm{ul} /} / v_{j}^{\mathrm{ul}}=2\left(\left\langle v_{j}^{\mathrm{u}}\left|V_{\mathrm{pv}}\left(q_{j}\right) / h c\right| v_{j}^{\mathrm{u}}\right\rangle-\left\langle v_{j}^{\mathrm{l}}\left|V_{\mathrm{pv}}\left(q_{j}\right) / h c\right| v_{j}^{1}\right\rangle\right) / \widetilde{x}_{j}^{\mathrm{ul}}
$$

where $v_{j}^{\mathrm{u}}$ denotes the perturbed upper vibrational level, $v_{j}^{1}$ the corresponding lower vibrational level and $\tilde{x}_{j}^{\text {ul }}$ is the wavenumber term difference between two levels in mode $j$. For vibrational fundamentals this is denoted $\tilde{v}_{j}$ (anharmonic) or $\tilde{\omega}_{j}$ (harmonic).

\subsection{Reverse adiabatic harmonic approximation for vibrational frequency shifts}

One may furthermore approximate the anharmonic vibrational fundamental frequency shift between enantiomers, $\Delta_{\mathrm{pv}} v_{j}$, by the shift of the harmonic frequencies arising from the apparent change of the effective force constants in the normal coordinate $q_{j}$ due to the parity violating potential:

$$
\begin{aligned}
& \tilde{\omega}_{j} \approx \tilde{\omega}_{j}^{0}+\Delta_{\mathrm{pv}} \tilde{\omega}_{j} \\
& \Delta_{\mathrm{pv}} \tilde{v}_{j} \approx \Delta_{\mathrm{pv}} \tilde{\omega}_{j}\left(\tilde{v}_{j} / \tilde{\omega}_{j}^{0}\right) \approx \frac{2}{h c} \cdot\left[l_{0}^{\dagger} F_{\mathrm{pv}} l_{0}\right]_{j j}\left(\tilde{v}_{j} / \tilde{\omega}_{j}^{0}\right)
\end{aligned}
$$

where $l_{0}$ diagonalizes the cartesian force constant matrix $F^{(x, m)}$ with eigenvalues related to $\widetilde{\omega}_{j}^{0}$. The factor 2 has been introduced because we consider the difference between enantiomers (denoted with $\Delta_{\mathrm{pv}}$, see Eq. (14)). In this approach we find

$$
\tilde{\omega}_{\mathrm{R}}-\tilde{\omega}_{\mathrm{S}}=\Delta_{\mathrm{pv}} \tilde{\omega}_{j} \approx 4 \cdot p_{2}(j)
$$

where the coordinate dependent ab initio parity violating potential $V_{\mathrm{pv}}$ has been fitted by a least-squares procedure to a polynomial expansion up to $n^{\text {th }}$ order

$$
\frac{V_{\mathrm{pv}}\left(q_{j}\right)}{h c}=\sum_{k=0}^{n} p_{k}(j) q_{j}^{k}
$$

In practice a third order expansion in Eq. (23) turns out to be sufficient.

This treatment actually corresponds to a model that interprets the parity violating effect as arising from a kinetic energy as well as from a potential energy contribution arising by effectively a reverse adiabatic approximation [45].

\subsection{Approximate theory of rotational frequency shifts and structural changes in polyatomic molecules due to parity violation}

\subsubsection{Structural changes}

The vibrational potential energy $V$ is modified by the parity violating potential $V_{\mathrm{pv}}$ and gives rise to a new global minimum which is shifted with 
respect to the minimum of the parity conserving potential (in reduced normal coordinates, the latter corresponds to $V\left(q_{1}=0, q_{2}=0, \cdots, q_{9}=0\right)$, which in internal coordinates corresponds to $V\left(r_{\mathrm{e}}^{0}(\mathrm{CH}), r_{\mathrm{e}}^{0}(\mathrm{CF}), r_{\mathrm{e}}^{0}(\mathrm{CCl})\right.$, $\left.r_{\mathrm{e}}^{0}(\mathrm{CBr}), \varphi_{\mathrm{e}}^{0}(\mathrm{HCF}), \varphi_{\mathrm{e}}^{0}(\mathrm{HCCl}), \varphi_{\mathrm{e}}^{0}(\mathrm{HCBr}), \varphi_{\mathrm{e}}^{0}(\mathrm{FCCl}), \varphi_{\mathrm{e}}^{0}(\mathrm{ClCBr})\right)$, determined without $V_{\mathrm{pv}}$ present. This change in equilibrium geometry induces a change of the rotational constants $A_{\mathrm{e}}, B_{\mathrm{e}}, C_{\mathrm{e}}$ which can be used to estimate the shifts on the pure rotational spectrum observable in the microwave region. Again, a general theory of the structure changes would require a minimum search on a $3 N-6$ dimensional potential energy hypersurface. This can be reduced by restriction to a more limited space, which can be chosen in several ways corresponding to different approximations. We discuss here in detail those results obtained with dimensionless reduced normal coordinates and refer more briefly to results in a non-redundant set of internal bond-lengths, bond-angles coordinates and other approximations when appropriate. For each vibrational mode (uncoupled, but due to $V_{\mathrm{pv}}$ no longer harmonic), we have a coordinate dependence (expressed in reduced normal coordinates of the molecular system without $V_{\mathrm{pv}}$ present) according to (see Eq. (23))

$$
\frac{V(q)}{h c}=\frac{1}{2} \tilde{\omega} q^{2}+\sum_{i=0}^{3} p_{k} q^{k} .
$$

The coordinate for the new minimum is approximately given by the expression

$$
q_{j \min } \approx-\frac{p_{1}(j)}{\widetilde{\omega}_{j}^{0}+2 p_{2}(j)} \approx-\frac{p_{1}(j)}{\widetilde{\omega}_{j}^{0}}
$$

which gives directly the shift with respect to $q_{j}=0$ and corresponds to a change in cartesian geometry. From this change of the cartesian geometry caused by $V_{\mathrm{pv}}$ we can also estimate a change of the bond lengths and bond angles. From the definition of the distance $r_{i j}$ between two atoms $i$ and $j$

$$
r_{i j}^{2}=\left(x_{i}-x_{j}\right)^{2}+\left(y_{i}-y_{j}\right)^{2}+\left(z_{i}-z_{j}\right)^{2}
$$

we obtain

$\Delta r_{i j} \approx \frac{\left(x_{i}^{0}-x_{j}^{0}\right)\left(\Delta x_{i}-\Delta x_{j}\right)+\left(y_{i}^{0}-y_{j}^{0}\right)\left(\Delta y_{i}-\Delta y_{j}\right)+\left(z_{i}^{0}-z_{j}^{0}\right)\left(\Delta z_{i}-\Delta z_{j}\right)}{r_{i j}^{0}}$

$r_{i j} \approx r_{i j}^{0}+\Delta r_{i j}$

by again neglecting nonlinear terms in $\Delta$. From the parity violating potential we thus have a linear perturbation $\Delta r_{i j}$ to the equilibrium bond length $r_{i j}^{0}$ (without $V_{\mathrm{pv}}$ present) which can be estimated by this procedure. The bond angle $\varphi$ between three atoms denoted 1-2-3 (with the central atom 2) is 
given by

$$
\begin{aligned}
\cos \left(\varphi^{0}+\Delta \varphi\right) \approx & \frac{\sum_{\underline{\alpha}=(x, y, z)}\left[\left(\alpha_{1}^{0}-\alpha_{2}^{0}\right)\left(\alpha_{3}^{0}-\alpha_{2}^{0}\right]\right.}{r_{21}^{0} r_{23}^{0}\left(1+\Delta r_{23} / r_{23}^{0}+\Delta r_{21} / r_{21}^{0}\right)} \\
& +\frac{\sum_{\alpha=(x, y, z)}\left[\left(\alpha_{1}^{0}-\alpha_{2}^{0}\right) \Delta \alpha_{23}+\left(\alpha_{3}^{0}-\alpha_{2}^{0}\right) \Delta \alpha_{21}\right]}{r_{21}^{0} r_{23}^{0}\left(1+\Delta r_{23} / r_{23}^{0}+\Delta r_{21} / r_{21}^{0}\right)} .
\end{aligned}
$$

We have to a reasonably good approximation for small $\Delta \varphi$ (for example, $y$ would correspond to $\Delta \varphi$ which is on the order of $10^{-15}$ )

$$
\begin{aligned}
& \arccos (x+y) \approx \arccos (x)+\arccos (y)-\frac{\pi}{2} \\
& \arccos (a \cdot y) \approx a \cdot \arccos (y)+\frac{\pi}{2}
\end{aligned}
$$

and consequently, the change of the bond angle, $\Delta \varphi$, in its linear approximation is

$$
\Delta \varphi \approx \arccos \left[\frac{\sum_{\alpha=(x, y, z)}\left[\left(\alpha_{1}^{0}-\alpha_{2}^{0}\right) \Delta \alpha_{23}+\left(\alpha_{3}^{0}-\alpha_{2}^{0}\right) \Delta \alpha_{21}\right]}{r_{21}^{0} r_{23}^{0}}\right] .
$$

The approximate expressions to determine $\Delta r$ and $\Delta \varphi$ have been checked by calculating the exact change from cartesian coordinates using high precision arithmetic from MAPLE [68] with 70 digits. The results differ by less than 10 percent.

\subsubsection{Changes in the moment-of-inertia tensor}

The (symmetric) moment-of-inertia tensor in the center-of-mass system $(\mathrm{CM})$ is given by [69]

$$
I=\left(\begin{array}{ccc}
I_{x} & -D_{x y} & -D_{x z} \\
-D_{x y} & I_{y} & -D_{y z} \\
-D_{x z} & -D_{y z} & I_{z}
\end{array}\right)
$$

where the diagonal element $I_{x}$ (and similarly $I_{y}, I_{z}$ ) is given by

$$
\begin{aligned}
I_{x} & =\sum_{\alpha} m_{\alpha}\left[\left(x_{\alpha, y}+\Delta x_{\alpha, y}\right)^{2}+\left(x_{\alpha, z}+\Delta x_{\alpha, z}\right)^{2}\right] \\
& \approx \sum_{\alpha} m_{\alpha}\left[x_{\alpha, y}^{2}+x_{\alpha, z}^{2}\right]+2 \sum_{\alpha} m_{\alpha}\left[x_{\alpha, y} \Delta x_{\alpha, y}+x_{\alpha, z} \Delta x_{\alpha, z}\right] \\
& \approx I_{x}^{0}+2 \sum_{\alpha} m_{\alpha}\left[x_{\alpha, y} \Delta x_{\alpha, y}+x_{\alpha, z} \Delta x_{\alpha, z}\right] \\
& \approx I_{x}^{0}+\Delta I_{x}
\end{aligned}
$$


and the off-diagonal element $D_{x y}$ (similarly for $D_{x z} D_{y z}$ ) by

$$
\begin{aligned}
D_{x y} & =\sum_{\underline{\alpha}} m_{\alpha}\left(x_{\alpha, x}+\Delta x_{\alpha, x}\right)\left(x_{\alpha, y}+\Delta x_{\alpha, y}\right) \\
& \approx D_{x y}^{0}+\sum_{\alpha} m_{\alpha}\left[x_{\alpha, x} \Delta x_{\alpha, y}+x_{\alpha, y} \Delta x_{\alpha, x}\right] \\
& \approx D_{x y}^{0}+\Delta D_{x y} .
\end{aligned}
$$

The summation is over the $N$ atoms, $\alpha=1 \cdots N$, and $\Delta x$ denotes the change in cartesian coordinates due to $V_{\mathrm{pv}}$. We neglected quadratic and higher terms in $\Delta f(f=D, I, x)$ since $\Delta f / f$ itself is on the order of $10^{-15}$. This form gives the change in the moment of inertia, $\Delta \mathbf{I}$ as a linear perturbation to $\mathbf{I}^{0}$ with a common scaling factor, and is therefore well suited for numerical evaluation again without any loss of precision. The cartesian change in geometry due to the shift in reduced normal coordinates is given by Eq. (8) in this approximation.

\subsubsection{Changes in the rotational constants}

The rotational constants $A_{\mathrm{e}}, B_{\mathrm{e}}, C_{\mathrm{e}}$ are inversely proportional to the eigenvalues of the inertial tensor, i.e.

$$
\begin{aligned}
& \mathbf{Z}^{\top} \mathbf{I} \mathbf{Z}=\operatorname{diag}\left(I_{A}, I_{B}, I_{C}\right) \\
& X_{\mathrm{e}}=\frac{h}{8 \pi^{2} c I_{X}}
\end{aligned}
$$

with $X=A, B, C$. Rigorously one has thus the zero order ("parity conserving") rotational constants from

$$
\mathbf{Z}_{0}^{\top} \mathbf{I}_{0} \mathbf{Z}_{0}=\operatorname{diag}\left(I_{A}^{0}, I_{B}^{0}, I_{C}^{0}\right)
$$

and the rotational constants including parity violation from

$$
\mathbf{Z}^{\top}\left(\mathbf{I}_{0}+\Delta \mathbf{I}\right) \mathbf{Z}=\operatorname{diag}\left(I_{A}^{0}+\Delta I_{A}, I_{B}^{0}+\Delta I_{B}, I_{C}^{0}+\Delta I_{C}\right) .
$$

Because $\Delta I_{A}, \Delta I_{B}, \Delta I_{C}$ are very small, one can use the following approximation to calculate the changes in rotational constants: We assume that the diagonal elements $I_{\mathrm{pv}}^{x x}$ (where $x$ labels the three diagonal elements as a,b,c) of $\mathbf{Z}_{0}^{\top} \Delta \mathbf{I} \mathbf{Z}_{0}$ provides an estimate for the change in the principal moments of inertia $\mathbf{I}_{X}^{0}$, where $\mathbf{Z}_{0}$ is the eigenvector matrix obtained by diagonalizing $\mathbf{I}^{0}$. If we denote the rotational constant obtained by diagonalizing $\mathbf{I}^{0}+\Delta \mathbf{I}$ by $X_{\mathrm{e}}$ following Eqs. (35), (36) we have

$$
\begin{aligned}
X_{\mathrm{e}} & \approx X_{\mathrm{e}}^{0}+\Delta X_{\mathrm{e}} \\
& \approx X_{\mathrm{e}}^{\mathrm{o}} \cdot\left(1-I_{\mathrm{pv}}^{x x} / I_{X}^{0}\right) .
\end{aligned}
$$

Thus we have

$$
\Delta X_{\mathrm{e}} / X_{\mathrm{e}}^{0}=\left(X_{\mathrm{e}} / X_{\mathrm{e}}^{0}\right)-1 \approx-X_{\mathrm{e}}^{\mathrm{o}} / X_{\mathrm{pv}}=-I_{\mathrm{pv}}^{x x} / I_{\mathrm{X}}^{0}
$$

where $X_{\mathrm{pv}}=h /\left(8 \pi^{2} c I_{\mathrm{pv}}^{x x}\right)$. Again, the approximate expressions have been 
checked with MAPLE [68] and no deviations where found for the values presented here. The microwave spectral changes are related to the changes of rotational energy levels. While there are no simple expressions for asymmetric tops (where all rotational constants are different), the leading terms in the energy level expressions can be estimated from expressions of the kind

$$
E_{J} \approx(h c) C_{0} J(J+1)+\cdots
$$

with total angular momentum quantum number $J$, and a selection rule $\Delta J= \pm 1$ transition wavenumbers are of the order

$$
\tilde{v} \approx 2 C_{0} J+\cdots
$$

and relative frequency shifts

$$
\frac{\Delta \tilde{v}_{\text {rot }}}{\tilde{v}_{\text {rot }}} \approx \frac{\Delta C_{0}}{C_{0}} .
$$

Here $C_{0}$ is the effective rotational constant in the vibrational ground state and $\Delta C_{0}$ the corresponding shift due to parity violation. These are not easily calculated, as they again require the solution of a multidimensional rovibrational Schrödinger equation [62]. However, consistent with other approximations we may use

$$
\frac{\Delta \tilde{v}_{\text {rot }}}{\tilde{v}_{\text {rot }}} \approx \frac{\Delta C_{0}}{C_{0}} \approx \frac{\Delta C_{\mathrm{e}}}{C_{\mathrm{e}}}
$$

where $\Delta C_{\mathrm{e}}$ and $C_{\mathrm{e}}$ are directly available from the above calculations. Exact solutions for rotational transitions (including even rovibrational couplings) would be possible in principle [70], but are hardly required at the present level of approximation. The changes $\Delta \mathbf{I}$ and $\Delta A(\Delta B, \Delta C)$ discussed in the present section 2.5 all refer to changes from the (R) enantiomer (which we used throughout the calculations, except when explicitely stated otherwise) when including the parity violating potential with respect to the parity conserving result. In order to obtain differences $\Delta_{\mathrm{pv}} X$ that exist between (R) and (S) enantiomers, $\Delta_{\mathrm{pv}}=\Delta^{(\mathrm{R})}-\Delta^{(\mathrm{S})}$, one may multiple $\Delta X$ by 2 , for example

$$
\Delta_{\mathrm{pv}} C_{\mathrm{e}}=\Delta C_{\mathrm{e}}^{(\mathrm{R})}-\Delta C_{\mathrm{e}}^{(\mathrm{S})} \approx 2 \Delta C_{\mathrm{e}}^{(\mathrm{R})} .
$$

This has also been verified numerically.

\section{Results and discussion}

$\mathrm{CHBrClF}$ is a chiral molecule which has already been investigated, both, spectroscopically and theoretically by high-resolution Fourier-Transform vibrational overtone spectroscopy in the infrared range for the isolated $\mathrm{CH}$ chromophore presented elsewhere [36-38, 71]. To determine the normal 
Table 1. Harmonic vibrational wave numbers $\omega$ in $\mathrm{cm}^{-1}$ for $\mathrm{CHBrClF}$; Basis 1: 6$311+\mathrm{G}(\mathrm{d}, \mathrm{p})$; Basis $2: 6-311+\mathrm{G}(2 \mathrm{~d}, 2 \mathrm{p})$; Basis 3: 6-311+G(2df,2pd); Basis 4: from refs. $[36,37,71]$ obtained with a basis set of essentially double-zeta quality; Basis 5 : VDZ [74]. Basis 4 has been used to determine the normal coordinates and Basis 5 has been used for $V_{\mathrm{pv}}$ calculations. The second column are anharmonic vibrational fundamental wavenumbers [37]. Total electronic energies $E_{\mathrm{tot}}$ are quoted as $E_{\mathrm{tot}} / E_{\mathrm{h}}=E_{\mathrm{MP} 2} / E_{\mathrm{h}}+3170$.

\begin{tabular}{lcccclc}
\hline & $v^{\exp }[37]$ & Basis 1 & Basis 2 & Basis 3 & $\begin{array}{l}\text { Basis 4 } \\
{[36,37,71]}\end{array}$ & Basis 5 \\
\hline$v_{1}$ & 3025.5 & 3197 & 3223 & 3195 & 3245 & 3211 \\
$v_{2}$ & 1306.2 & 1354 & 1341 & 1337 & 1375 & 1290 \\
$v_{3}$ & 1202.8 & 1278 & 1242 & 1243 & 1315 & 1229 \\
$v_{4}$ & 1077.2 & 1092 & 1085 & 1107 & 1098 & 1032 \\
$v_{5}$ & 787.0 & 833 & 796 & 820 & 838 & 708 \\
$v_{6}$ & 663.6 & 684 & 669 & 684 & 680 & 619 \\
$v_{7}$ & 425.2 & 438 & 428 & 436 & 437 & 373 \\
$v_{8}$ & 313.0 & 324 & 319 & 323 & 327 & 300 \\
$v_{9}$ & 223.6 & 237 & 229 & 231 & 240 & 217 \\
$E_{\text {to }} / E_{\mathrm{h}}$ & & -0.903291 & -0.983025 & -1.096614 & -0.124477 & 0.404182 \\
\hline
\end{tabular}

coordinates, we used the vibrational force field $F^{(x, m)}$ obtained at the level of second order Møller-Plesset perturbation theory (MP2) with basis 1 from our previous investigations [37]. This basis set is of double-zeta quality. We evaluated the coordinate dependence of $V_{\mathrm{pv}}\left(q_{j}\right)$ for wavefunctions from Hartree-Fock theory (RHF, [28]), the random-phase approximation (RPA, [30]) and complete-active space SCF (CASSCF, [30]), the latter only for some selected vibrational modes. Except for the RHF wavefunction, the other high quality wavefunctions have been used together with the multiconfiguration linear-response approach (MC-LR) described in detail elsewhere [30]. The results for vibrational frequencies from parity conserving potentials are summarized in Table 1. The parameters of the least-squares fit to describe the coordinate dependence of the parity violating potentials are listed in Table 2 for the nine vibrational modes.

\subsection{Harmonic vibrational effects induced by parity violation}

Table 1 summarizes the calculated harmonic vibrational frequencies obtained from MP2 calculations using different basis sets. The normal coordinates obtained from basis 4 have been used to determine the potential energy surface of the $\mathrm{CH}$ chromophore [37]. They define the reduced normal coordinate space for the present study of the parity violating potential. We use basis 5 for the pure electronic part of the $a b$ initio calculation of the parity violating potential.

The dependence of the parity violating potential $V_{\mathrm{pv}}\left(q_{j}\right)$ of (R)-CHBrClF on reduced normal coordinates is presented in Fig. 3 for all vibrational de- 
Table 2. Polynomial fit coefficients $p_{\mathrm{i}}$ and root-mean-square deviation $d_{\text {rms }}$ (all in $10^{-15} \mathrm{~cm}^{-1}$ ) for $v_{1}$ to $v_{9}$ obtained with $\overline{\mathrm{RPA}}$ (random-phase approximation) for the (R)-CHBrClF enantiomer. In parenthesis, the numerical uncertainty due to the least-squares procedure is given in units of the last significant digits.

\begin{tabular}{llrrrl}
\hline Mode & \multicolumn{1}{l}{$p_{0}$} & \multicolumn{1}{l}{$p_{1}$} & \multicolumn{1}{l}{$p_{2}$} & \multicolumn{1}{c}{$p_{3}$} & $d_{\text {rms }}$ \\
\hline 1 & $958.679(47)$ & $33.219(91)$ & $-6.746(26)$ & $-0.488(29)$ & 0.118 \\
2 & $958.062(446)$ & $680.124(869)$ & $114.879(251)$ & $8.010(274)$ & 1.120 \\
3 & $958.441(463)$ & $1175.441(903)$ & $-98.752(261)$ & $-2.099(284)$ & 1.163 \\
4 & $958.799(31)$ & $-294.061(60)$ & $16.791(17)$ & $0.1333(189)$ & 0.077 \\
5 & $958.754(7)$ & $331.877(14)$ & $-62.857(4)$ & $0.799(5)$ & 0.019 \\
6 & $958.772(13)$ & $-180.438(25)$ & $20.187(7)$ & $-1.345(8)$ & 0.033 \\
7 & $958.720(21)$ & $637.852(41)$ & $7.890(12)$ & $-0.447(13)$ & 0.053 \\
8 & $958.791(25)$ & $839.356(48)$ & $-4.811(14)$ & $0.396(15)$ & 0.062 \\
9 & $958.707(30)$ & $-166.116(59)$ & $-2.315(17)$ & $-0.125(18)$ & 0.075 \\
\hline
\end{tabular}

grees of freedom. We have plotted the one-dimensional functions of $V_{\mathrm{pv}}\left(q_{j}\right)$ from RPA in the reduced normal coordinate range $-2 \leq q_{j} \leq+2$. This can be converted to a normal coordinate range for $Q_{j}$ with $Q_{j} /[\AA \sqrt{\mathrm{u}}] \approx$ $q_{j} \cdot \sqrt{33.71527 /\left[\tilde{\omega}_{j} / \mathrm{cm}^{-1}\right]}$.

The variation of $V_{\mathrm{pv}}$ with $q_{j}$ is qualitatively similar for both, the RHF and the RPA calculations, however, there are quantitative differences which can be seen from the results listed in Table 3 .

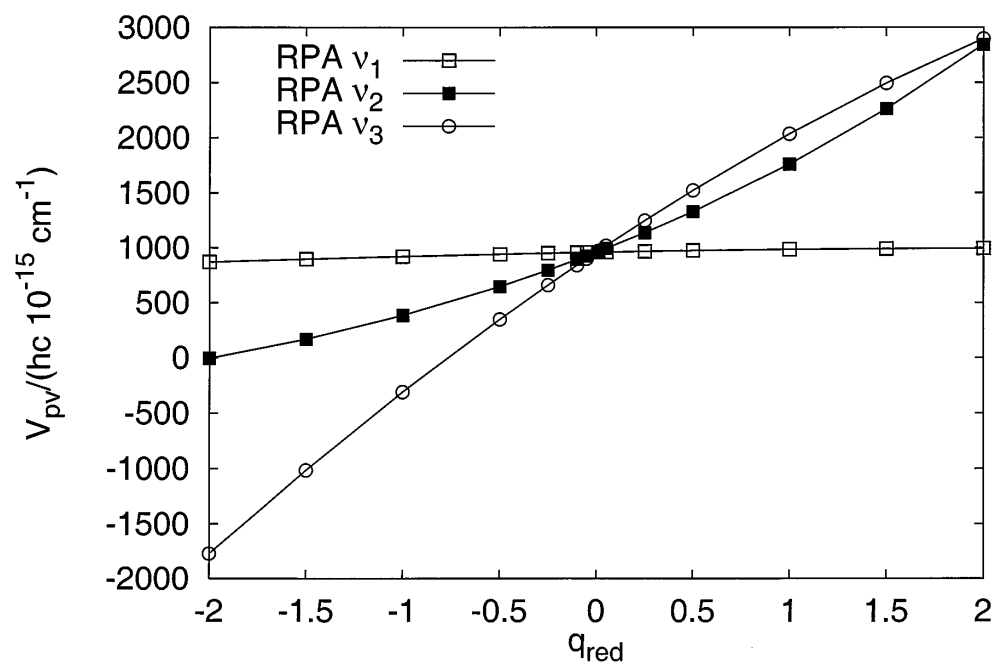

Fig. 3A 


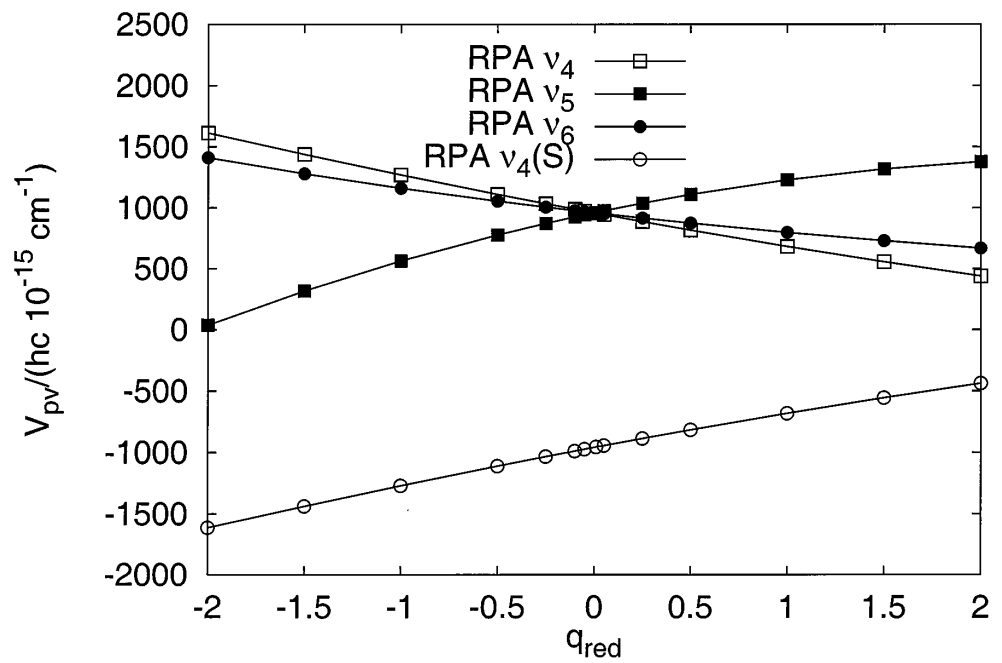

Fig. 3B

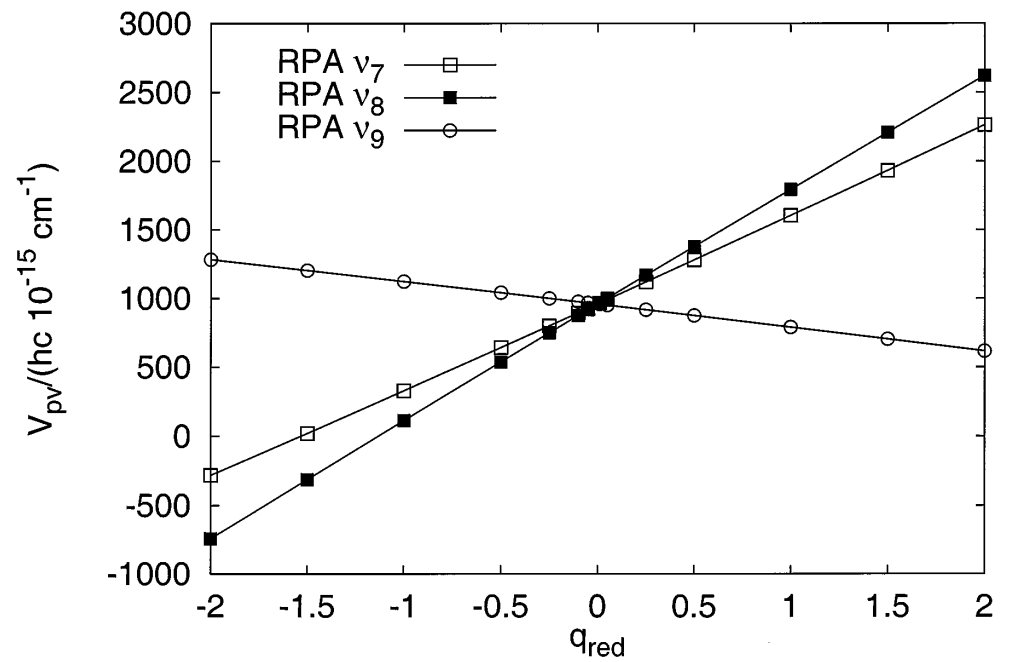

Fig. 3C

Fig. 3a-c. Dimensionless reduced normal coordinate dependence $\left(q_{\mathrm{red}}\right)$ of the parity violating potential $V_{\mathrm{pv}}\left(q_{\mathrm{red}}\right) / h c$ in units of $10^{-15} \mathrm{~cm}^{-1}$ for three different vibrational modes: (a) $v_{1}$ (open squares), $v_{2}$ (filled squares), $v_{3}$ (open circles), (b) $v_{4}$ (open squares), $v_{5}$ (filled squares), $v_{6}$ (filled circles), $v_{4}^{(S)}$ (open circles), and (c) $v_{7}$ (open squares), $v_{8}$ (filled squares), $v_{9}$ (open circles). All potentials have been calculated within the random-phase approximation (RPA) and for (R)-CHBrClF, except in (b) where we have also plotted the calculated parity violating potential of (S)-CHBrClF for $v_{4}^{(\mathrm{S})}$ (open circles) which shows the sign changes in $V_{\mathrm{pv}}$ for different enantiomers. 
The parity violating potential energy is almost constant over the whole $q$-range for the highest vibrational mode $v_{1}$, corresponding to the $\mathrm{CH}$ stretching vibration. Except for $q_{4}, q_{6}$ and $q_{9}$, all functions decrease with increasing reduced normal coordinate (for the phase convention see [36$38]$ ). The bending modes $v_{2}, v_{3}(\mathrm{CH}$ bend $), v_{7}$ (ClCF bend) and $v_{8}(\mathrm{BrCF}$ bend) reach comparably large $V_{\mathrm{pv}}$ values for strong elongation along $q$ (see Fig. 3). We have additionally calculated $V_{\mathrm{pv}}$ for the (S)-CHBrClF enantiomer. The fit gives, within numerical accuracy, the same magnitude but different signs for $V_{\mathrm{p} w}$ as expected. $\Delta_{\mathrm{pv}} E / h c$ at the equilibrium position is just given by $2 \cdot p_{0}$ which in case of RPA is about $873.68 \times 10^{-20} \mathrm{E}_{\mathrm{h}} \approx$ $1917.5 \times 10^{-15} \mathrm{hc} \mathrm{cm}^{-1}$. The fits give the same values of $p_{0}$ for all modes within the uncertainties of the fits (see Table 2).

Fig. 4 shows a plot of the scaled parity violating potential (multiplied by a factor of $3 \times 10^{-4}$, see Fig. $3 \mathrm{a}$ and $3 \mathrm{~b}$ ) for the CF stretching mode $v_{4}$ (Fig. 4a) and the FCBr bending mode $v_{8}$ (Fig. $4 \mathrm{~b}$ ). A first set of data is represented by the filled squares, the line represents the polynomial fit function (differences are invisible at this scale) to these data. We then calculated a second set of data in the range corresponding to an extrapolation. The empty circles represent these separate results which lie well on the cautiously extrapolated fit, the quality of which is thus confirmed to be excellent. We show also the vibrational anharmonic wavefunction for the vibrational ground state (dotted line) and the first excited state (solid line). These wavefunctions from the anharmonic adiabatic diagonal approximation are to scale and normalized to one. An analysis of the normal coordinates of $\mathrm{CHBrClF}$ according to the potential energy distribution [72] $P(i, j)=F(i, i) \cdot l^{2}(i, j) / \sum_{i}\left[F(i, i) \cdot l^{2}(i, j)\right]$ shows, that the lowest vibrational mode is a heavy mixture of the internal coordinates for $\mathrm{C}-\mathrm{Br}$ stretch and the angles between $\mathrm{H}-\mathrm{C}-\mathrm{Br}, \mathrm{F}-\mathrm{C}-\mathrm{Cl}$, and $\mathrm{F}-\mathrm{C}-\mathrm{Br}$. However, many of the other modes have a relatively local character, such as $v_{1}$ for $\mathrm{CH}$ stretching, $v_{2}, v_{3}$ for $\mathrm{CH}$ bending and $v_{4}$ for $\mathrm{CF}$ stretching (see also [37]). Table 2 collects the polynomial fit parameters for the one dimensional parity violating potentials obtained with RPA and basis 5 .

The second derivatives of the force constant matrix needed to determine $\Delta_{\mathrm{pv}} \omega$ according to Eqs. (20) and (21) have been obtained by a fit of $V_{\mathrm{pv}}(q)$ data obtained in the reduced normal coordinate range from -2 to +2 to a third order analytical polynomial expression with a least squares procedure. The quadratic term $p_{2}$ is a measure for the perturbation of the force constant by the parity violating potential. The root-mean-square value of the fit to 13 data points is slightly better than $10^{-16} \mathrm{~cm}^{-1}$. The values obtained for the two $\mathrm{CH}$ bending modes ( $v_{2}$ and $v_{3}$ ) have the largest uncertainties. All other parameters are statistically very well determined.

Within the reverse adiabatic, harmonic approximation, the largest relative shift in the harmonic vibrational frequencies due to the parity violating potential, as presented in Table 3, is obtained for the $\mathrm{CH}$-bending and the 

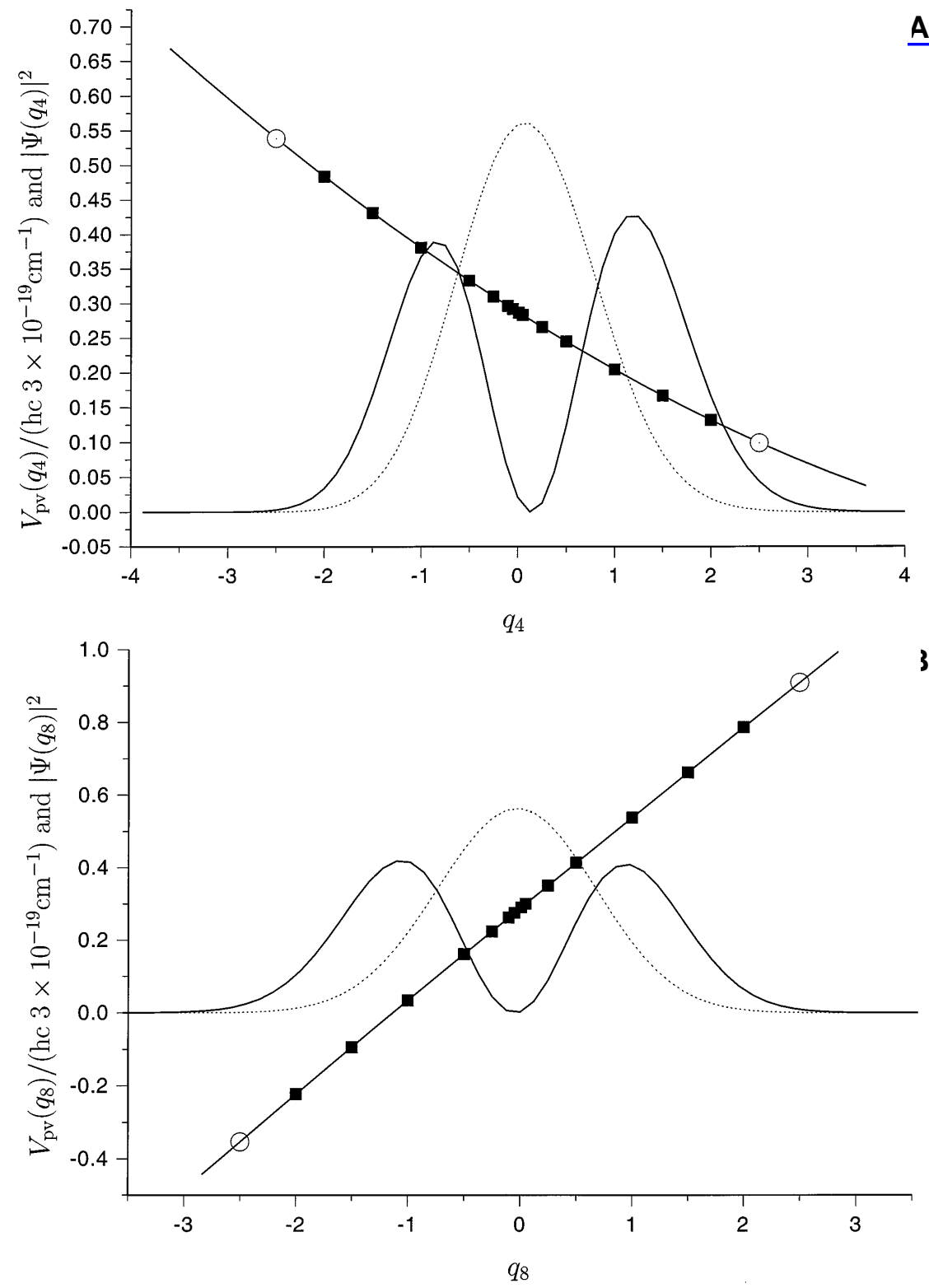

Fig. 4. Calculated $V_{\mathrm{pv}}\left(q_{\mathrm{red}}\right)$ (points) from Fig. 3 (additionally multiplied by $3 \times 10^{-4}$ for graphical representation) and the vibrational anharmonic one dimensional wavefunction for the vibrational ground state (dotted line) and first excited state (solid line) of (a) $v_{4}$ and (b) $v_{8}$. The filled squares correspond to the first set of ab initio data, the line is fitted by a least-squares procedure to a third order polynomial (see text), and the potential values at -2.5 and +2.5 (open circles) which lie outside the fitted range from -2.0 to +2.0 have been obtained from a separate $a b$ initio calculation and thus confirm the extrapolated prediction from the fit. 
Table 3. Harmonic vibrational wavenumbers $\widetilde{\omega}$ (in $\mathrm{cm}^{-1}$ ) and relative frequency shifts $\Delta_{\mathrm{pv}} \tilde{\omega} / \tilde{\omega}$ in the reserve harmonic approximation (see Eq. $(22)$ ). The second column are anharmonic vibrational fundamental wavenumbers [71]. The third column provides harmonic frequencies from refs. [36, 37, 71] obtained with a basis set of essentially doublezeta quality.

\begin{tabular}{llrrr}
\hline$j$ & $\tilde{v}_{j}^{\text {exp }} / \mathrm{cm}^{-1}$ & $\omega_{j} / \mathrm{cm}^{-1}$ & \multicolumn{2}{c}{$\frac{\Delta_{\mathrm{pv}} \omega_{j}}{\omega_{j}} / 10^{-19}$} \\
\cline { 3 - 4 } & & & $\mathrm{RHF}$ & \multicolumn{1}{c}{$\mathrm{RPA}$} \\
\hline 137,71$]$ & 3026 & 3245.000 & -13.9 & -83.2 \\
2 & 1306 & 1375.227 & +1134.0 & +3341.4 \\
3 & 1203 & 1315.363 & -1173.3 & -3003.1 \\
4 & 1077 & 1097.851 & +348.9 & +611.8 \\
5 & 787 & 838.309 & -1613.6 & -2999.2 \\
6 & 664 & 680.406 & +385.4 & +1186.8 \\
7 & 425 & 437.436 & -498.5 & +721.4 \\
8 & 313 & 327.435 & +490.9 & -587.7 \\
9 & 224 & 240.356 & -150.5 & -385.2 \\
\hline
\end{tabular}

CCl-stretching vibrations. This applies to both calculations presented here, namely the sum-over-states expression in the RHF approach, as well as to the linear-response approximation in the RPA approach. Because the effect is so small (note the scaling of $10^{-19}$ for the relative shift, as given in Table 3), a direct experimental observation of the shift $\Delta \omega / \omega$ on the order of $10^{-16}$ is difficult (and so far unsuccessful [35-43]).

This, however, does not render the attempt to look for experimental evidence of the parity violating potential in vibrational spectra of molecules a priori hopeless. It has been discussed before that some excitation schemes to "achiral" levels of well defined parity might be a better approach to the signatures of parity violating effects in molecular systems [16, 17, 38, 73] (see also Fig. 1).

\subsection{Role of the $a b$ initio calculation}

Table 4 shows the parameters from the fit of $V_{\mathrm{pv}}$ for the CF-stretching mode $\left(v_{4}\right)$ to the third order polynomial Eq. (23) for RPA and three high quality MC-LR(CASSCF) wavefunctions by steadily increasing the active space from 8 electrons distributed in 10 orbitals (denoted $[8,10]$ ) to finally 12 electrons in 12 orbitals $([12,12])$, which is already a fairly large active space. The energy difference between the (R)- and the (S)-CHBrClF, $\Delta_{\mathrm{pv}} E$ / $h c$, is related to $2 \cdot p_{0}$, and the corresponding harmonic frequency shift $\Delta_{\mathrm{pv}} \widetilde{\omega}$ to $4 \cdot p_{2}$ in the reverse adiabatic harmonic approximation. The RPA seems to be a relatively good approximation. We also compare to the RHF-SDE 
Table 4. Polynomial fit coefficients $p_{i}$ and root-mean-square deviation $d_{\mathrm{rms}}$ (all in $10^{-15} \mathrm{~cm}^{-1}$ ) for $v_{4}$ obtained with different $a b$ initio methods, namely RPA (random-phase approximation) and MC-LR CASSCF (multi-configuration linear response completeactive space $\mathrm{SCF}$ ) for the (R)-CHBrClF enantiomer. In parenthesis, the numerical uncertainty due to the least-squares procedure is given in units of the last significant digits.

\begin{tabular}{rrlrrr}
\hline \multicolumn{1}{l}{ Method } & \multicolumn{1}{l}{$p_{0}$} & \multicolumn{1}{c}{$p_{1}$} & \multicolumn{1}{c}{$p_{3}$} & \multicolumn{1}{c}{$d_{\text {rms }}$} \\
\hline RHF & $38.872(5)$ & $-135.762(9)$ & $9.575(2)$ & $-0.1171(29)$ & 0.012 \\
RPA & $958.799(31)$ & $-294.061(60)$ & $16.791(17)$ & $0.1333(189)$ & 0.077 \\
{$[8,10]$ CAS } & $799.209(28)$ & $-258.520(58)$ & $18.656(17)$ & $-0.1128(184)$ & 0.076 \\
{$[8,12]$ CAS } & $783.629(76)$ & $-265.083(149)$ & $19.474(43)$ & $0.0704(468)$ & 0.192 \\
{$[12,12]$ CAS } & $962.698(45)$ & $-246.650(87)$ & $14.706(25)$ & $0.0822(275)$ & 0.113 \\
\hline
\end{tabular}

approach in the first line of Table 4, which shows that the deficiency of RHF-SDE is substantial for $p_{0}$ (i.e. $\Delta_{\mathrm{pv}} E$ ), as shown by us before for other molecules [27-30]. The effect on the higher coefficients $p_{1}$ to $p_{3}$, which determine the vibrational shift, is smaller.

\subsection{Anharmonic effects}

The harmonic model of calculating the frequency shift caused by the parity violating energy difference is approximate. A severe approximation is to neglect anharmonic contributions. From our investigation of the isolated $\mathrm{CH}$-chromophore we know that diagonal and off-diagonal anharmonicity has a pronounced influence on the vibrational band positions and wavefunctions. A three-dimensional vibrational analysis based on ab initio potential energy surfaces revealed a strong anharmonic Fermi-resonance coupling between the $\mathrm{CH}$-stretching motion and the two $\mathrm{CH}$-bending motions. The relatively poor fit of $V_{\mathrm{pv}}$ for those modes $(1-3$, see Table 2) might be an indication that this coupling is also of some influence on $V_{\mathrm{pv}}$. We discuss here first the diagonal anharmonic contribution to the parity violation induced frequency shift. This is achieved with $1^{\text {st }}$ order perturbation theory by calculating the expectation value $\left\langle v^{\prime}\left|V_{\mathrm{pv}}\right| v^{\prime}\right\rangle$ using anharmonic vibrational wavefunctions as discussed in section 2 . The results are collected in Table 5.

The harmonic values in Table 5 have been obtained by calculating the expectation value with a harmonic vibrational potential energy. These harmonic shifts are a factor of 2 smaller than those reported in Table 3 , as can be easily understood. Calculating the parity violation induced frequency shift from the force constants (see above) implies that the parity violation Hamiltonian is interpreted as a Hamiltonian with a kinetic and a potential energy. In case of a harmonic oscillator, the virial theorem connects the expectation value of the kinetic energy $\langle T\rangle$ with the expectation value of the 
Table 5. Relative vibrational frequency shifts from expectation values (in $10^{-19}$ ) according to Eq. (19) between (R)- and (S)-CHBrClF enantiomers. The parity violating potential is from RPA calculations (Table 2). (a) tabulated as $2\left(\left\langle 1\left|\tilde{V}_{\mathrm{pv}}\right| 1\right\rangle-\left\langle 0\left|\tilde{V}_{\mathrm{pv}}\right| 0\right\rangle\right) / \tilde{\omega}_{1}$ based on harmonic vibrational wavefunctions for the ground and first vibrationally excited state, $|0\rangle$ and $|1\rangle$; (b) tabulated as $2\left(\left\langle 1^{\prime}\left|\tilde{V}_{\mathrm{pv}}\right| 1^{\prime}\right\rangle-\left\langle 0^{\prime}\left|\tilde{V}_{\mathrm{pv}}\right| 0^{\prime}\right\rangle\right) / \tilde{v}_{1}$ based on anharmonic vibrational wavefunctions for the ground and first vibrationally excited state, $\left|0^{\prime}\right\rangle$ and $\left|1^{\prime}\right\rangle$.

\begin{tabular}{|c|c|c|c|}
\hline \multirow[t]{2}{*}{ Mode $\mathrm{j}$} & \multicolumn{2}{|c|}{$\Delta_{\mathrm{pv}} v_{j}^{1 \leftarrow 0} / v_{j}^{1 \leftarrow 0}$} & \multirow[t]{2}{*}{ Note } \\
\hline & $\operatorname{harmonic}^{(a)}$ & anharmonic $^{(\mathrm{b})}$ & \\
\hline 1 & -41.6 & 8.4 & this work \\
\hline 2 & 1670.7 & 1319.2 & this work \\
\hline 3 & -1501.5 & -1287.7 & this work \\
\hline \multirow[t]{2}{*}{4} & 305.9 & -805.9 & this work \\
\hline & 500.4 & -623.0 & [47] \\
\hline \multirow[t]{2}{*}{5} & -1499.6 & -2499.5 & this work \\
\hline & -1853.1 & -3028.5 & [47] \\
\hline \multirow[t]{2}{*}{6} & 593.4 & 952.9 & this work \\
\hline & 186.3 & 522.0 & [47] \\
\hline 7 & 360.7 & 1809.5 & this work \\
\hline 8 & -293.8 & -3360.7 & this work \\
\hline 9 & -192.6 & 450.1 & this work \\
\hline
\end{tabular}

potential energy $\langle V\rangle$ according to

$$
\langle T\rangle=\langle V\rangle
$$

and this explains the factor of two difference between the calculation of the frequency shift in the reverse adiabatic harmonic approximation and the adiabatic harmonic approximation as expectation value of the potential energy. Some of the shifts have recently also been calculated in the relativistic Dirac Fock framework [47]. As can be seen from Tables 5 and 3, the relativistic corrections are modest even for $\mathrm{CHBrClF}$, containing $\mathrm{Br}$ as rather heavy atom. The harmonic Dirac Fock results in Table 5 should be compared to the harmonic RHF results in Table 3 (divided by 2). The corrections are modest, and no larger than the changes which result from going beyond the Hartree Fock level and from including anharmonicity.

We furthermore observe a strong dependence of the shift on anharmonic contributions. Table $6 \mathrm{a}$ and $6 \mathrm{~b}$ offer detailed insight into the cause for the remarkable changes. Table 6 a summarizes the expectation values for the ground state $\langle 0|\cdots| 0\rangle$ and the first excited state $\langle 1|\cdots| 1\rangle$ of the CFstretching mode $v_{4}$, and similarly Table $6 \mathrm{~b}$ for $v_{8}$. The second column in Table 6 are the polynomial parameters $p_{i}$ (in $10^{-15} \mathrm{~cm}^{-1}$, see Table 2). The expectation values for each individial polynomial term $p_{i} \cdot q^{i}(i=0,1,2$, $3)$ are listed separately. For harmonic oscillator wavefunctions the expectation value for odd powers of $q$ is exactly zero and the quadratic term 
Table 6a. Term-by-term analysis of expectation values contributing to the frequency shift in $v_{4}$ as listed in Table 5 (see Eq. (19)) with anharmonic and harmonic vibrational wavefunctions for the vibrational ground and the first excited state (see also caption to Table 5).

\begin{tabular}{|c|c|c|c|}
\hline$i$ & $p_{i}$ & $\left\langle 0\left|p_{i} \cdot q^{i}\right| 0\right\rangle$ & $\left\langle 1\left|p_{i} \cdot q^{i}\right| 1\right\rangle$ \\
\hline \multicolumn{4}{|c|}{ Anharmonic wavefunctions } \\
\hline $\begin{array}{l}0 \\
1 \\
2 \\
3 \\
\text { Sum }\end{array}$ & $\begin{array}{r}958.80 \\
-294.06 \\
16.79 \\
0.13\end{array}$ & $\begin{array}{r}958.80 \\
-31.02 \\
8.68 \\
0.03 \\
936.49\end{array}$ & $\begin{array}{r}958.80 \\
-93.29 \\
27.27 \\
0.17 \\
892.95\end{array}$ \\
\hline \multicolumn{4}{|c|}{ Harmonic wavefunctions } \\
\hline $\begin{array}{l}0 \\
1 \\
2 \\
3 \\
\text { Sum }\end{array}$ & $\begin{array}{r}958.80 \\
-294.06 \\
16.79 \\
0.13\end{array}$ & $\begin{array}{r}958.80 \\
0.00 \\
8.40 \\
0.00 \\
967.19\end{array}$ & $\begin{array}{r}958.80 \\
0.00 \\
25.19 \\
0.00 \\
983.99\end{array}$ \\
\hline
\end{tabular}

Table 6b. Term-by-term analysis of expectation values contribution to the frequency shift in $v_{8}$ as listed in Table 5 (see Eq. (19)) with anharmonic and harmonic vibrational wavefunctions for the ground and the first excited state (see also caption to Table 5). This bending vibration shows the largest absolute shift, taking anharmonic contributions into account

\begin{tabular}{|c|c|c|c|}
\hline$i$ & $p_{i}$ & $\left\langle 0\left|p_{i} \cdot q^{i}\right| 0\right\rangle$ & $\left\langle 1\left|p_{i} \cdot q^{i}\right| 1\right\rangle$ \\
\hline \multicolumn{4}{|c|}{ Anharmonic wavefunctions } \\
\hline $\begin{array}{l}0 \\
1 \\
2 \\
3 \\
\text { Sun }\end{array}$ & $\begin{array}{r}958.79 \\
839.36 \\
-4.81 \\
0.40\end{array}$ & $\begin{array}{r}958.79 \\
-30.35 \\
-2.41 \\
-0.03 \\
926.00\end{array}$ & $\begin{array}{r}958.79 \\
-80.33 \\
-7.27 \\
-0.15 \\
871.05\end{array}$ \\
\hline \multicolumn{4}{|c|}{ Harmonic wavefunctions } \\
\hline $\begin{array}{l}0 \\
1 \\
2 \\
3 \\
\text { Sun }\end{array}$ & $\begin{array}{r}958.79 \\
839.36 \\
-4.81 \\
0.40\end{array}$ & $\begin{array}{r}958.79 \\
0.00 \\
-2.41 \\
0.00 \\
956.40\end{array}$ & $\begin{array}{r}958.79 \\
0.00 \\
-7.21 \\
0.00 \\
951.48\end{array}$ \\
\hline
\end{tabular}

increases with vibrational excitation. The contributions responsible for the strong difference between expectation values calculated from harmonic and anharmonic wavefunctions are the odd terms, particularly $p_{1}$. The absolute value of the anharmonic matrix elements of $p_{1}$ and $p_{3}$ increases with vibrational excitation. 
Table 7. Anharmonic relative vibrational frequency shifts $\Delta v / v$ from expectation values (in $10^{-19}$ ) according to Eq. (19) between (R)- and (S)-CHBrClF enantiomers for overtone transitions between the vibrational ground state (denoted 0) and the second, third and fourth vibrationally excited state for some modes $v_{2}$ ( $\mathrm{CH}$ bending), $v_{4}$ (CF stretching), $v_{5}$ (CCl stretching) and $v_{6}(\mathrm{CBr}$ stretching $)$. The parity violating potential is from RPA calculations (Table 2).

\begin{tabular}{lllll}
\hline Mode & $v_{2}$ & $v_{4}$ & $v_{5}$ & $v_{6}$ \\
\hline Transition & & & $\left(\boldsymbol{\Delta}_{\mathrm{pv}} v / v\right) / 10^{-16}$ & \\
\hline $2 \leftarrow 0$ & +1.3389 & -0.7806 & -2.5382 & +0.5934 \\
$3 \leftarrow 0$ & +1.3527 & -0.7587 & -2.5799 & +0.5934 \\
$4 \leftarrow 0$ & +1.3547 & -0.7492 & -2.6177 & +0.5934 \\
\hline
\end{tabular}

\subsection{Effects on overtone transitions}

In Table 7 we summarize some vibrational frequency shifts for overtones of $v_{2}(\mathrm{CH}$ bending $), v_{4}(\mathrm{CF}$ stretching $), v_{5}(\mathrm{CCl}$ stretching $)$ and $v_{6}(\mathrm{CBr}$ stretching).

Some modes show an increase of the relative anharmonic frequency shift (see Eq. (19)) upon overtone excitation (e.g., $\left.v_{2}\right)$ whereas for $v_{6}(\mathrm{CBr}$ stretching) overtone excitation does not influence the relative shift at all. So far there is no obvious trend which would make a prediction possible without explicitely calculating the expectation values. It should be clear as well, that at higher vibrational excitations the one dimensional separable anharmonic approximation is unreliable and thus the present results are at best rough estimates.

\subsection{Equilibrium structural effects and rotational frequency shifts}

In Table 8 we collected the change in bond lengths and bond angles caused by parity violation. There is no obvious relation between the deviation of a bond length from its equilibrium value and the vibrational frequency shift. The approximate relation connecting the cartesian coordinate change with a change in bond angle, as derived above (column I, Table 8), is in good agreement with the exact value obtained by using MAPLE V [68] with high-precision arithmetic (column II). Following a different, more approximate route than presented here, the effect of parity violation on the molecular structure has been discussed in an early paper [25]. The parity violating effect on vibrational frequencies has therein been based on a discussion using internal bond-angle and bond-lengths coordinates thereby assuming that bond length and angles are parity-even whereas torsional and dihedral angles are parity-odd. It is clear, however, that this classification is erroneous in that parity transformation properties are connected only to car- 
Table 8. Change in internal coordinates due to $V_{\mathrm{pv}}(\vec{q})$ for the (R)-CHBrClF enantiomer determined in dimensionsless reduced normal coordinates. (I) Obtained with MAPLE V [68]; (II) obtained from the approximate expressions (see text for discussion); (III) obtained from (diagonal) $V_{\mathrm{pv}}(\vec{r})$ determined in internal coordinates (see text for discussion). The definitions are $\Delta r_{\mathrm{e}}=r_{\mathrm{e}}\left(V+V_{\mathrm{pv}}\right)-r_{\mathrm{e}}(V)$ and $\Delta \varphi_{\mathrm{e}}=\varphi_{\mathrm{e}}\left(V+V_{\mathrm{pv}}\right)-\varphi_{\mathrm{e}}(V)$.

\begin{tabular}{|c|c|c|c|c|c|}
\hline & \multirow{3}{*}{$\begin{array}{l}\text { Equilibrium } \\
{[36,37,71]}\end{array}$} & \multicolumn{4}{|l|}{ Perturbed } \\
\hline & & \multicolumn{3}{|l|}{ RPA } & \multirow{2}{*}{ RHF } \\
\hline & & $\mathrm{I}$ & II & III & \\
\hline & $r / \AA$ & \multicolumn{4}{|c|}{$\Delta r /\left(10^{-19} \AA\right)$} \\
\hline$r(\mathrm{CH})$ & 1.08065 & 0.70 & 0.70 & -4.84 & 5.0 \\
\hline$r(\mathrm{CF})$ & 1.36085 & 198 & 198 & 181 & 68 \\
\hline$r(\mathrm{CCl})$ & 1.75285 & -397 & -397 & -366 & -205 \\
\hline \multirow[t]{2}{*}{$r(\mathrm{CBr})$} & 1.92202 & 62 & 62 & 178 & 44 \\
\hline & $\varphi /$ degrees & \multicolumn{4}{|c|}{$\Delta \varphi /\left(10^{-15}\right.$ degrees $)$} \\
\hline$\varphi(\mathrm{HCF})$ & 108.431 & -5.70 & -5.15 & -2.1 & -1.48 \\
\hline$\varphi(\mathrm{HCBr})$ & 108.289 & -4.99 & -4.69 & -4.2 & -2.70 \\
\hline$\varphi(\mathrm{HCCl})$ & 108.507 & 12.76 & 11.77 & 11.2 & 5.84 \\
\hline$\varphi(\mathrm{FCCl})$ & 109.024 & -8.21 & -7.94 & -4.6 & -5.03 \\
\hline$\varphi(\mathrm{ClCBr})$ & 113.382 & -2.23 & -2.50 & -1.7 & -0.06 \\
\hline
\end{tabular}

tesian coordinates. An estimate for the equilibrium deviation in bond length on the order of $10^{-20} \AA$ was presented [25], which is one to three orders of magnitude smaller than the results shown in Table 8. Our approach, however, is more consistent and extends the "independent mode approximation" briefly discussed in [25]. Column III in Table 8 summarizes those internal coordinates changes that are obtained by calculating the parity violating potential in a non-redundant set of internal coordinates. This set of internal coordinates consists of the following bond lengths and bond angles: $r(\mathrm{CH})$, $r(\mathrm{CF}), r(\mathrm{CCl}), r(\mathrm{CBr}), \varphi(\mathrm{HCF}), \varphi(\mathrm{HCCl}), \varphi(\mathrm{HCBr}), \varphi(\mathrm{FCCl}), \varphi(\mathrm{ClCBr})$. They differ by two angles from those used in [37] to determine the harmonic force field. This choice has been made to facilitate an additional test of our results (see below). From the cartesian mass-weighted force constant matrix $\mathbf{F}^{(x, m)}$ we obtain the internal coordinates force constant matrix $\mathbf{F}^{\text {int }}$ with the use of the contravariant tensor (Wilson's $B$-matrix) [58]

$$
B_{k, i}=\frac{\partial S_{k}}{\partial x_{i}}
$$

that transforms between cartesian $\left(x_{i}\right)$ and internal $\left(S_{k}\right)$ coordinates in matrix notation as

$$
\begin{aligned}
& \mathbf{F}^{\mathrm{int}}=\mathbf{A}^{\top} \mathbf{F}^{(x, m)} \mathbf{A} \\
& \mathbf{A}=\mathbf{M}^{-1} \mathbf{B}^{\top}\left(\mathbf{B} \mathbf{M}^{-1} \mathbf{B}^{\top}\right)^{-1} .
\end{aligned}
$$


Table 9. Rotational constants $\underline{A}_{0}, B_{0}, C_{0}$ from experiment [35], $A_{\mathrm{e}}, B_{\mathrm{e}}, C_{\mathrm{e}}$ calculated $a b$ initio [37] and calculated relative change of rotational constants $\Delta_{\mathrm{pv}} X_{\mathrm{e}} / X_{\mathrm{e}}^{0}=2 \Delta X_{\mathrm{e}} / X_{\mathrm{e}}^{0} \approx$ $-2 X_{\mathrm{e}}^{\mathrm{0}} / X_{\mathrm{pv}}$ (see Eqs. (40) and (45)) with $X=A, B, C$ due to parity-violating effects for RPA. There are no differences between the values in the last column and those obtained by diagonalizing the complete moment-of-inertia tensor using high precision arithmetic (MAPLE V, [68]), see text. Note that $\Delta X_{\mathrm{e}}^{(R)}=-\Delta X_{\mathrm{e}}^{(S)}$, which has been verified numerically (see text).

\begin{tabular}{llll}
\hline & Exp. [35] & ab initio (this work) & $\Delta_{\mathrm{pv}} X_{\mathrm{e}} /\left(X_{\mathrm{e}}^{\mathrm{o}} \cdot 10^{-18}\right)$ \\
\hline$A / \mathrm{cm}^{-1}$ & $0.215711541(23)$ & 0.21802 & 32.84 \\
$B / \mathrm{cm}^{-1}$ & $0.067998430(14)$ & 0.06704 & 11.31 \\
$C / \mathrm{cm}^{-1}$ & $0.053396944(16)$ & 0.05292 & 20.34 \\
\hline
\end{tabular}

M denotes a $3 N \times 3 N$ diagonal matrix with the atomic masses. B is not a square matrix and can thus not be inverted. The cartesian force constant matrix is taken from ab initio calculations [37]. We have also carried out calculation with a nonseparable, explicitly coupled parity violating potential in the subspace of the three internal coordinates $r(\mathrm{Cl}), r(\mathrm{CBr}), \varphi(\mathrm{CClBr})$. The results are similar to those in column III (Table 8), however with sizeable changes in a few instances.

Table 9 summarizes the relative change of the $A, B, C$ rotational constants between both enantiomers caused by $V_{\mathrm{pv}}$. This change is on the order of $10^{-17}$. The separation of corresponding rotational transitions in both enantiomers is proportional to $\Delta_{\mathrm{pv}} X=2 \Delta X$ and the rotational quantum number $J$. It should be made clear that because of the various approximations, the result for $\Delta_{\mathrm{pv}} X$ in Table 9 must be considered order of magnitude estimates, not accurate predictions of rational line shifts, which would require inclusion of the anharmonic vibrational wave function [62].

\section{Conclusions}

From the present investigation we can draw the following main conclusions:

(i) The complete theory of parity violating rovibrational frequency differences between enantiomers of chiral molecules requires the solution of complicated multidimensional anharmonically and rovibrationally coupled problems. However, a first approximation is possible by reducing the problem to a set of one dimensional integrals over the parity violating potentials in terms of the molecular dimensionless reduced normal coordinate $q_{i}$.

(ii) The parity violating potentials can be expanded to an excellent approximation as a low order Taylor expansion. 
(iii) The first term $p_{0}\left(=\Delta_{\mathrm{pv}} E / 2\right)$ is equal to half the parity violating energy difference between enantiomers and is much larger when calculated with MC-LR (CASSCF and RPA) approaches as compared to the traditional SDE-RHF approaches (more than a factor of 20 smaller for $\mathrm{CHBrClF}$ ). This confirms our similar findings on the deficiency of SDE-RHF (on other molecules) which used both CIS and MC-LR approaches to prove this effect [27-30]. For $\mathrm{CHBrClF}$ the (S) enantiomer is calculated to be more stable than $(\mathrm{R})$ by about $10^{-11} \mathrm{~J} \mathrm{~mol}^{-1}$.

(iv) The higher terms of the Taylor expansion of $V_{\mathrm{pv}}\left(q_{i}\right)$ govern the vibrational frequency shifts. They depend somewhat less strongly on the level of calculation (RHF versus MC-LR CASSCF and RPA). However, they do depend very strongly on the vibrational mode considered. The relative frequency shifts $\Delta_{\mathrm{pv}} v / v$ fall in the range of $10^{-(16 \pm 1)}$ for CHBrClF.

(v) One finds a substantial difference between harmonic (both adiabatic and reverse adiabatic) and one dimensional anharmonic approximations for calculating the vibrational frequency shifts.

(vi) The exact calculation of structural changes leads again to very complicated multidimensional problems, which can be reduced to lower dimensionality only approximately. Different routes in this approximation lead to somewhat different predictions of structural changes, which are however of similar order of magnitude. Differences between RHF and RPA results are sizeable.

(vii) Using the structural changes with parity violation to predict differences between rotational frequencies of $(\mathrm{R})$ and $(\mathrm{S})$ enantiomers one finds values $\Delta v / v$ between $10^{-17}$ and $10^{-16}$.

The present results show that all current experiments are still orders of magnitude away from the accuracy needed to achieve an observation of parity violating frequency shifts between (R)- and (S)-CHBrClF, say for the well analysed $v_{4}$ band $[35,41,42]$. On the other hand, the strong vibrational mode dependence demonstrated here, together with the expected increase of the effects with heavy atom substitution, indicate that experimental observation of parity violating effects in rovibrational transitions may soon be possible. For such experiments, the careful prediction of the most suitable vibrational mode in a suitable molecule using the theoretical methods developed here should be helpful. However, even observation of these rovibrational frequency shifts will not provide the most important quantity, $\Delta_{\mathrm{pv}} E$, as discussed in connection with Fig. 1. For this purpose, the kind of experiment proposed in $[16,17]$ with intermediate levels of preferred parity is necessary. For these more difficult experiments, the present calculations show a very large increase in predicted $\Delta_{\mathrm{pv}} E$ compared to earlier expectations, which should render the effect more easily observable. Current work 
is in progress in our laboratory towards experiments on suitable molecules as well as towards extending theory to treat more accurately the multidimensional rovibrational problems.

\section{Acknowledgements}

Substantial help from and discussions with Robert Berger are gratefully acknowledged as well as early advice (in spring 1998) by Peter Taylor on the DALTON program and discussions with Wim Klopper on relativistic effects (in summer 1999) and a recent preprint of [47] on these qustions by Peter Schwerdtfeger.

Our work is supported financially by ETH Zürich (including computer support through C4, Cray and CSCS) and the Schweizerischer Nationalfonds.

\section{References}

1. T. D. Lee and C. N. Yang, Phys. Rev. 104 (1956) 254.

2. C. S. Wu, R. W. Hayward, D. D. Hoppes and R. P. Hudson, Phys. Rev. 105 (1957) 1413.

3. S. Glashow, Nucl. Phys. 22 (1961) 579.

4. S. Weinberg, Phys. Rev. Lett. 19 (1967) 1264.

5. A. Salam, in Proceedings of the Eighth Nobel Symposium (edited by N. Svartholm), Amkvist and Wiksell, Stockholm (1968) 367.

6. R. E. Novick, Thirty years of parity nonconservation, Birkhäuser, Basel (1988).

7. M. Veltman. In: The Rise of the Standard Model, L. Hoddeson et al. (eds.) Cambridge U.P., Cambridge (1977) 145.

8. M.-A. Bouchiat and C. Bouchiat, Rep. Prog. Phys. 60 (1997) 1351

9. I. B. Khriplovich, Parity Nonconservation in Atomic Phenomena. Gordon and Breach, Philadelphia (1991).

10. Y. Yamagata, J. Theoret. Biol. 11 (1966) 495.

11. D. Rein, J. Mol. Evol. 4 (1974) 15.

12. V. Letokhov, Phys. Lett. A53 (1975) 275.

13. B. Zel'dovich, Sov. Phys. JETP 9 (1959) 682.

14. B. Zel'dovich, D. Saakyan and I. Sobel'man, JETP Lett. 25 (1977) 94.

15. R. Harris and L. Stodolski, J. Chem. Phys. 73 (1980) 3862.

16. M. Quack, Angew. Chem. Int. Ed. Engl. 28 (1989) 571.

17. M. Quack, Chem. Phys. Lett. 132 (1986) 147.

18. M. Quack, Nova Acta Leopoldina, NF 81 (1999) 137.

19. D. W. Rein, R. A. Hegstrom and P. G. H. Sandars, Phys. Lett. A71 (1979) 499.

20. R. A. Hegstrom, D. W. Rein and P. G. H. Sandars, J. Chem. Phys. 73 (1980) 2329.

21. S. F. Mason and G. E. Tranter, Chem. Phys. Lett. 94 (1983) 34.

22. S. F. Mason and G. E. Tranter, Mol. Phys. 53 (1984) 1091.

23. G. E. Tranter, Chem. Phys. Lett. 115 (1985) 286.

24. G. E. Tranter, Chem. Phys. Lett. 120 (1985) 93.

25. G. E. Tranter, Chem. Phys. Lett. 121 (1985) 339

26. A. McDermott and G. Tranter, Chem. Phys. Lett. 163 (1989) 1.

27. A. Bakasov, T. K. Ha and M. Quack. In J. Chela-Flores and F. Rolin, editors, Proc. of the $4^{\text {th }}$ Trieste Conference (1995), Chemical Evolution: Physics of the Origin and Evolution of Life, pages 287-296, Kluwer Academic Publishers, Dordrecht (1996). 
28. A. Bakasov, T. K. Ha and M. Quack, J. Chem. Phys. 109 (1998) 7263; ibid. 110 (1999) 6081.

29. A. Bakasov and M. Quack, Chem. Phys. Lett. 303 (1999) 547.

30. R. Berger and M. Quack, J. Chem. Phys. 112 (2000) 3148.

31. P. Lazzeretti and R. Zanasi, Chem. Phys. Lett. 279 (1997) 349.

32. R. Zanasi and P. Lazzeretti, Chem. Phys. Lett. 286 (1998) 240.

33. J. Laerdahl and P. Schwerdtfeger, Phys. Rev. A 60 (1999) 4439.

34. A. Barra, J. Robert and L. Wiesenfeld, Phys. Lett. A 115 (1986) 443.

35. A. Bauder, A. Beil, D. Luckhaus, F. Müller and M. Quack, J. Chem. Phys. 106 (1997) 7558.

36. A. Beil, D. Luckhaus, R. Marquardt and M. Quack, J. Chem. Soc. Faraday Discuss. 99 (1994) 49.

37. A. Beil, D. Luckhaus and M. Quack, Ber. Bunsenges. Phys. Chem. 100 (1996) 1853.

38. A. Beil, D. Luckhaus, M. Quack and J. Stohner, Ber. Bunsenges. Phys. Chem. 101 (1997) 311

39. O. Kompanets, A. Kukudzhanov, V. Letokhov and L. Gervits, Opt. Commun. 19 (1976) 414

40. E. Arimondo, P. Glorieux and T. Oka, Opt. Commun. 23 (1977) 369.

41. C. Daussy, Thèse de doctorat, Paris (1999).

42. C. Daussy, T. Marrel, A. Amy-Klein, C. Nguyen, C. Bordé and C. Chardonnet, Phys. Rev. Lett. 83 (1999) 1554.

43. H. Hollenstein, D. Luckhaus, J. Pochert, M. Quack and G. Seyfang, Angew. Chemie Intl. Ed. 36 (1997) 140.

44. R. Compton, unpublished work, cited in R. F. Service, Science 286 (1999) 1282.

45. J. Stohner, A. Beil, H. Hollenstein, O. Monti and M. Quack. In $37^{\text {th }}$ IUPAC Congress and $27^{\text {th }}$ GDCh Meeting, Berlin, Germany, August 14-19, 1999, Frontiers in Chemistry: Molecular Basis of the Life Sciences, page 525. ISBN 3-924763-82-8; and M. Quack and J. Stohner, Phys. Rev. Lett. (2000) in press.

46. M. Quack and J. Stohner, in SASP 2000 Proc. of the XIIth Symposium on Atomic and Surface Physics and related topics, January 30-February 5 (2000) eds. Davide Bassi and Paolo Tosi, Costa di Folgaria Universita di Trento (Italia) 2000.

47. J. K. Laerdahl, P. Schwerdtfeger and H. M. Quiney, Phys. Rev. Lett. (2000) in press.

48. J. H. van't Hoff, Vorlesungen über theoretische und physikalische Chemie, Band 2, Vieweg, Braunschweig (1899); Die Lagerung der Atome im Raume, Vieweg, Braunschweig (1876); in B. M. Bazendijk (Ed.): La chimie dans l'espace, Rotterdam (1887).

49. P. J. Mohr and B. N. Taylor, J. Phys. Chem. Ref. Data 27 (1999).

50. M. Bouchiat and C. Bouchiat, J. Physique 35 (1974) 899.

51. C. Cohen-Tannoudji, B. Diu and F. Lalöe, Quantum Mechanics. Vol. II, John Wiley, New York (1977)

52. S. Fraga and G. Malli, Many Electron Systems: Properties and Interactions, W. B. Saunders Co., Philadelphia (1968). R. R. Ernst, G. Bodenhausen and A. Wokann, Principles of Nuclear Magnetic Resonance in One and Two Dimensions, Oxford, Clarendon Press.

53. H. Kiyonaga, K. Morihashi and O. Kikuchi, J. Chem. Phys. 108 (1998) 2041.

54. M. J. Frisch, G. W. Trucks, M. Head-Gordon, P. M. W. Gill, M. W. Wong, J. B Foresman, B. G. Johnson, H. B. Schlegel, M. A. Robb, E. S. Replogle, R. Gomperts, J. L. Andres, K. Ragavachari, J. S. Binkley, C. Gonzales, R. L. Martin, D. J. Fox, D. J. Defrees, J. Baker, J. J. P. Stewart and J. A. Pople, Gaussian 94, Gaussian Inc., Pittsburgh PA (1994).

55. H. Ågren, O. Vahtras and B. Minaev, Adv. Quant. Chem. 27 (1996) 71.

56. T. Helgacker, H. Jensen, P. Joergensen, J. Olsen, K. Ruud, H. Ågren, T. Andersen, K. Bak, V. Bakken, O. Christiansen, P. Dahle, E. Dalskov, T. Enevoldsen, B. Fernan- 
dez, H. Heiberg, H. Hettema, D. Jonsson, S. Kirpekar, R. Kobayashi, H. Koch, K. Mikkelsen, P. Norman, M. Packer, T. Saue, P. Taylor and O. Vahtras, DALTON, an Electronic Structure Program, release 1.0 (1997).

57. M. Quack, Annu. Rev. Phys. Chem. 41 (1990) 839.

58. E. B. Wilson Jr., J. C. Decius and P. C. Cross, Molecular vibrations. The theory of infrared and Raman vibrational spectra. McGraw-Hill Inc., New York (1955).

59. J. Murrell, S. Carter, S. Farantos, P. Huxley and A. Varandas, Molecular Potential Energy Functions, John Wiley, New York (1984).

60. M. Quack, Phil. Trans. R. Soc. Lond. A332 (1990) 203.

61. M. Quack, J. Mol. Struct. 292 (1993) 171.

62. H. Hollenstein, R. Marquardt, M. Quack and M. A. Suhm, J. Chem. Phys. 101 (1994) 3588.

63. M. Quack and M. A. Suhm, Spectroscopy and quantum dynamics of hydrogen fluoride clusters, in J. Bowman and Z. Bačić, editors, Advances in Molecular Vibration and Collision Dynamics, Vol. III Molecular Clusters, pages 205-248, JAI Press (1998).

64. R. Marquardt and M. Quack, J. Chem. Phys. 109 (1998) 10628.

65. R. Meyer, J. Chem. Phys. 52 (1970) 2053.

66. D. Luckhaus and M. Quack, Chem. Phys. Lett. 190 (1992) 581.

67. A. Beil, H. Hollenstein, O. Monti, M. Quack and J. Stohner, J. Chem. Phys. in press (2000).

68. B. Char, K. Geddes, G. Gonnet, B. Leong, M. Monagan and S. Watt, Maple V. Waterloo Maple Software, Waterloo, Ontario (1990).

69. H. W. Kroto, Molecular Rotation Spectra, John Wiley, New York, first edition (1975).

70. D. Luckhaus and M. Quack, Mol. Phys. 68 (1989) 745.

71. A. Beil, D. Luckhaus, R. Marquardt and M. Quack, J. Chem. Soc. Faraday Discuss. 99 (1994) 96.

72. Y. Morino and K. Kuchitsu, J. Chem. Phys. 20 (1952) 1809.

73. M. Quack, Chapter 27 in "Femtosecond Chemistry" ed. by J. Manz and L. Woeste, Vch Publishers, Weinheim (1995) 781-817.

74. A. Schäfer, H. Horn and R. Ahlrichs, J. Chem. Phys. 97 (1992) 2571. 